\title{
Feasibility Studies on Pyro-SFR Closed Fuel Cycle and Direct Disposal of Spent Nuclear Fuel in Line with the Latest National Policy and Strategy of Korea
}

\author{
Muhammad Minhaj Khan, Jae Min Lee, Jae Hak Cheong, and Joo Ho Whang \\ Kyung Hee University, 1732 Deogyeong-daero, Giheung-gu, Yongin-si, Gyeonggi-do 17104, Republic of Korea \\ Correspondence should be addressed to Jae Hak Cheong; jhcheong@khu.ac.kr
}

Received 20 June 2017; Revised 22 August 2017; Accepted 17 September 2017; Published 31 October 2017

Academic Editor: Arkady Serikov

Copyright (C) 2017 Muhammad Minhaj Khan et al. This is an open access article distributed under the Creative Commons Attribution License, which permits unrestricted use, distribution, and reproduction in any medium, provided the original work is properly cited.

\begin{abstract}
With a view to providing supportive information for the decision-making on the direction of the future nuclear energy systems in Korea (i.e., direct disposal or recycling of spent nuclear fuel) to be made around 2020, quantitative studies on the spent nuclear fuel (SNF) including transuranic elements (TRUs) and a series of economic analyses were conducted. At first, the total isotopic inventory of TRUs in the SNF to be generated from all thirty-six units of nuclear power plants in operation or under planning is estimated based on the Korean government's official plan for nuclear power development. Secondly, the optimized deployment strategies are proposed considering the minimum number of sodium cooled-fast reactors (SFRs) needed to transmute all TRUs. Finally, direct disposal and Pyro-SFR closed nuclear energy systems were compared using equilibrium economic model and considering reduction of TRUs and electricity generation as benefits. Probabilistic economic analysis shows that the assumed total generation cost for direct disposal and Pyro-SFR closed nuclear energy systems resides within the range of 13.60 33.94 mills $/ \mathrm{kWh}$ and 11.40 25.91 mills/kWh, respectively. Dominant cost elements and the range of SFR overnight cost which guarantees the economic feasibility of the PyroSFR closed nuclear energy system over the direct disposal option were also identified through sensitivity analysis and break-even cost estimation.
\end{abstract}

\section{Introduction}

As of March 2016, the amount of spent nuclear fuel (SNF) produced from twenty units of pressurized light water reactors (PWRs) and four units of pressurized heavy water reactors (PHWRs) was reported to be about 14,608 metric tons of uranium (MTU) from the whole twenty-four units of Korean nuclear power plants (NPPs) (i.e., PWRs and PHWRs) [1]. The generation of SNF will continually increase since Korea is officially planning to deploy an additional eight units of Advanced Power Reactor 1400 (APR-1400) and four units of Advanced Power Reactor Plus (APR+) type PWRs by 2029 as scheduled in the 7th Basic Plan on Electricity Demand and Supply promulgated in July 2015 [2].

Due to the limited capacities of the SNF storage facilities at existing NPPs sites, the Korean government established the Basic Plan on High-level Radioactive Waste Management in July 2016, which specifies the plan for operation of a centralized SNF interim storage facility and a high-level radioactive waste (HLW) disposal facility by 2035 and 2053, respectively [3].

In parallel, the Korean government also plans to decide whether or not to commercialize the Pyro-SFR (pyroprocessing-sodium cooled-fast reactor) fuel cycle technology which has been developed since 1997, by around 2020, based upon the result of ongoing feasibility studies as clearly addressed in the Strategy for Technical Development and Demonstration of Future Nuclear Energy System published in July 2016 [4]. Accordingly, the national direction of the back-end fuel cycle is to be decided in the next few years.

Feasibilities of direct disposal and Pyro-SFR fuel cycle options based upon the national policy and strategies may provide an important input for the Korean government in deliberating optimized fuel cycle directions. A number of 


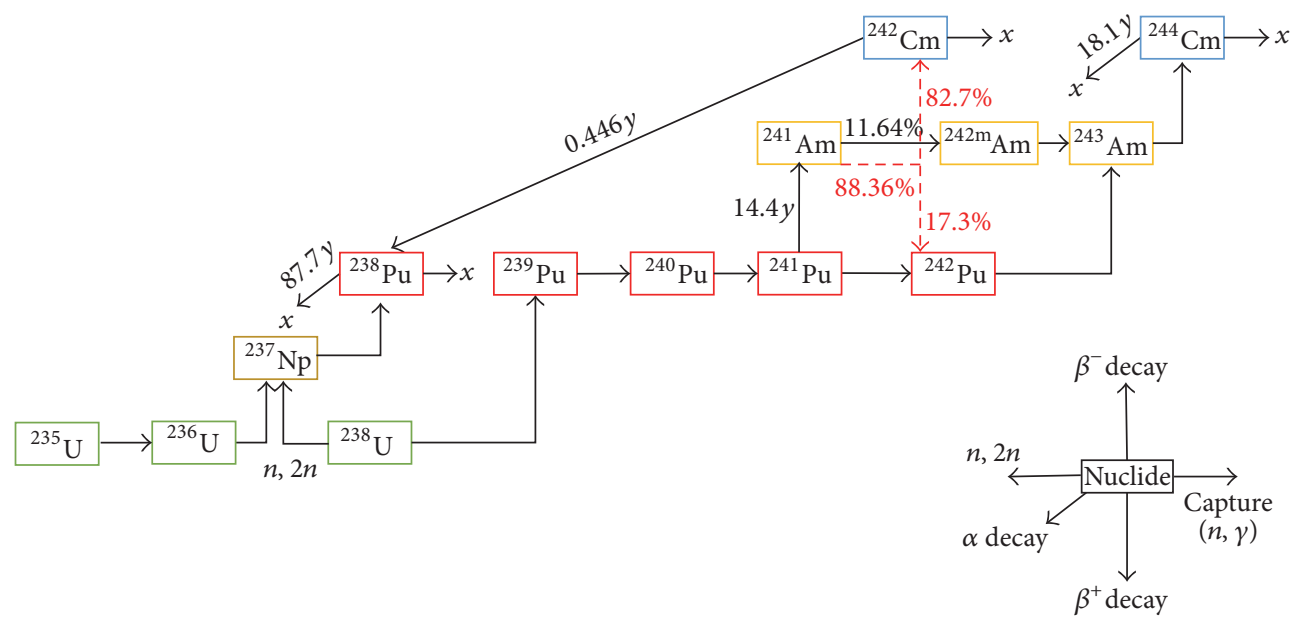

Note. The symbol " $x$ " represents the point at which the transmutation of a radionuclide through a specific reaction is not expected or its contribution is negligible.

Figure 1: Simplified transmutation model adopted in CAIN [25].

quantitative and economic studies have been reported on the back-end of fuel cycle options for Korea [5-21]. However, most of the previous studies were conducted based upon the assumptions proposed by the investigators rather than upon the official national policy $[6-8,14-16]$. In addition, neither detailed estimation of the total amount of SNF and TRUs in terms of radioactivity nor relative radiological toxicities of the SNF to be produced from all units of Korean NPPs have been openly reported. Though a few investigators have calculated the anticipated cost for a nuclear fuel cycle in Korea, no detailed economic feasibility studies have been reported on the overall costs and benefits from open or closed fuel cycle nuclear energy systems being considered in Korea [11-17]. In this context, the electricity generation from SFRs as well as NPPs (i.e., PWRs and PHWRs) could be considered as a potential benefit from the fuel cycle.

Thus, the main objective of this study is set to support the decision-making on the future nuclear fuel cycle options in Korea to be made by around 2020 through providing the following information:

(i) Total amount of SNF and TRUs $\left({ }^{237} \mathrm{~Np},{ }^{241} \mathrm{Am}\right.$, ${ }^{242 \mathrm{~m}} \mathrm{Am},{ }^{243} \mathrm{Am},{ }^{242} \mathrm{Cm}$, and ${ }^{244} \mathrm{Cm}$ and other plutonium isotopes such as ${ }^{238} \mathrm{Pu},{ }^{239} \mathrm{Pu},{ }^{240} \mathrm{Pu},{ }^{241} \mathrm{Pu}$, and ${ }^{242} \mathrm{Pu}$ ) to be accumulated in accordance with the latest national policy.

(ii) Time-dependent variations of radiotoxicity due to TRUs in SNF in the long term.

(iii) Optimal deployment strategies of SFRs to transmute the total inventory of TRUs efficiently.

(iv) Economic feasibilities on the overall costs and benefits of open or closed fuel cycle options using probabilistic economic analysis.

\section{Materials and Methodology}

2.1. Calculation of Maximum Inventory of SNF and Radioactivity of TRUs. In this study, the Nuclear Fuel Cycle Simulation
System (NFCSS) developed by the International Atomic Energy Agency (IAEA) was used to estimate the amount of SNF and TRUs from Korean NPPs. The NFCSS is a scenariobased computer model widely used for quantitative studies on various nuclear fuel cycle scenarios [22-24].

A fuel depletion model known as Calculation Actinide Inventory (CAIN) to perform isotopic depletion and decay calculations to solve Bateman's equations for a point assembly using one group neutron cross section as shown in (1) was adopted in the NFCSS.

$$
\begin{aligned}
\frac{d N_{i}}{d t}= & -\sum_{j}\left[\lambda_{i}^{i \rightarrow j}+\sigma_{i}^{i \rightarrow j} \bar{\varphi}\right] N_{i} \\
& +\sum_{j \neq i}\left[\lambda_{j}^{j \rightarrow i}+\sigma_{j}^{j \rightarrow i} \varphi\right] N_{j},
\end{aligned}
$$

where $N_{i}$ and $N_{j}$ are the atomic contents of isotopes $i$ and $j$, respectively; $\lambda_{i}^{i \rightarrow j}$ and $\lambda_{j}^{j \rightarrow i}$ are the decay constants of nuclides $i$ and $j$ which decay to $i(1 / \mathrm{s})$, respectively; $\sigma_{i}^{i \rightarrow j}$ and $\sigma_{j}^{j \rightarrow i}$ are the transmutation cross sections from isotope $i$ to $j$ (barn) and from isotope $j$ to $i$ (barn), respectively; and $\varphi$ is the average neutron flux $\left(\mathrm{n} /\left(\mathrm{s} \cdot \mathrm{cm}^{2}\right)\right)$.

Figure 1 shows the transmutation chains implemented in CAIN of the NFCSS to calculate the isotopic composition of SNF $[25,26]$.

First, calculation was done by using the NFCSS to estimate the generation of SNF including isotopes of plutonium $\mathrm{Pu}$ ) and MAs (minor actinides) from twenty-four units of NPPs in operation as of March 2016. As eight units of APR1400 and four units of APR+ type PWRs will be added by the end of 2029, future estimation for the generation of the SNF from thirty-six units of NPPs by considering their initial design lifetimes (i.e., 30, 40, 60, and 60 years for PHWR, PWR, APR-1400, and APR+, resp.) was made. As the thirtysixth NPP $(\mathrm{APR}+)$ will reach its initial design lifetime of sixty years by the end of 2089, estimation of the inventory of SNF 
and TRUs has been made by the year of 2089 as a reference year [2].

In order to predict the maximum inventory of SNF and TRUs to be produced from all Korean NPPs, twenty years of continued operations of NPPs after termination of their initial design lifetimes were additionally assumed [8]. However, Kori Unit 1 whose initial design lifetime has been already extended for ten years (i.e., from 2007-06-19 to 2017-06-18) was shut down permanently on June 19, 2017, in accordance with the government policy [2]. In addition, the second term of continued operation of 10 years for Wolsong Unit 1 is assumed, of which its first continued operation until 2022-11-20 was approved in 2015. As the thirty-sixth NPP $(\mathrm{APR}+)$ will reach its extended design lifetime by the end of 2109 , the potential maximum inventory of SNF and TRUs was estimated by the year of 2109 as another reference year.

Table 1 shows the full list of all units of Korean NPPs (PWRs, PHWRs) which have been already deployed or will be deployed by 2029, along with the time of commercial operation and termination of initial and extended design lifetime [27].

The time-dependent material flow of SNF for each NPP throughout its lifetime is calculated by MS Excel ${ }^{\mathrm{TM}}$ spreadsheet based upon the basic information on the Korean NPPs as shown in Table 1 and the calculation results of the NFCSS such as the amount of SNF including isotopic composition of TRUs to be generated from each Korean NPP per cycle.

2.2. Calculation of Nominal Radiotoxicities. In order to quantify the radiological hazard from each nuclide of TRUs and/or overall hazard from multiple nuclides, a few nominal expressions of radiotoxicity or radiotoxicity index for SNF were reported [28]. In this study, the time-variant nominal radiotoxicity index (in $\mathrm{Sv}$ or $\mathrm{Sv} / \mathrm{g}$ ) of SNF was proposed as

$$
\begin{gathered}
\text { Radiotoxicity Index }=\sum_{i} A_{i} \cdot e^{-\lambda_{i} t} \cdot \mathrm{EDC}_{\mathrm{ing}, i} \\
\text { or } \sum_{i} C_{i} \cdot e^{-\lambda_{i} t} \cdot \mathrm{EDC}_{\mathrm{ing}, i} \\
A_{i}=\lambda_{i} \cdot N_{i}=\frac{\ln 2}{T_{1 / 2, i}} \cdot m_{i} \cdot \frac{N_{A}}{M_{i}},
\end{gathered}
$$

where $A_{i}$ is the radioactivity of a TRU nuclide $i(\mathrm{~Bq}), C_{i}$ is the radioactivity concentration of nuclide $i(\mathrm{~Bq} / \mathrm{g}), \mathrm{EDC}_{\mathrm{ing}, i}$ is the effective dose coefficient to an adult age group for ingestion (Sv/Bq), $\lambda_{i}$ is the decay constant of nuclide $i(1 / \mathrm{y}), t$ is the elapsed time from discharge of the SNF from reactor core $(y)$, $N_{i}$ is the number of nuclides $i, m_{i}$ is the mass of nuclide $i(\mathrm{~g})$, $N_{A}$ is Avogadro's number $\left(6.02 \times 10^{23} / \mathrm{mol}\right), M_{i}$ is the atomic mass of TRU nuclide $i(\mathrm{~g} / \mathrm{mol})$, and $T_{1 / 2, i}$ is the half-life of TRU nuclide $i(\mathrm{y})$.

Only ingestion rather than inhalation pathway is considered in (2), since SNF is to be disposed of in deep geological media under direct disposal scenario and the volatilities of the actinides are low enough [29]. The values for $A_{i}$ and $C_{i}$ in (2) can be obtained from the nuclide-specific total inventory of TRUs in Bq and total mass of SNF calculated by use of the NFCSS in terms of grams. The values of $\mathrm{EDC}_{\mathrm{ing}, i}$ can be found in a volume of the official publications of the International Commission on Radiological Protection (ICRP) [30].

The radiotoxicity of SNF is known to be controlled by fission products for the first 300 years after being discharged from the reactor core, but long-lived actinides contribute most to the radiotoxicity after 300 years [29]. Therefore, this study mainly focuses on the ingestion radiotoxicity indices for long-lived actinides rather than those for fission products.

2.3. Establishment of Strategic Scenarios for Transmutation of TRUs by SFRs. In order to compare the Pyro-SFR fuel cycles with direct disposal options and further to find optimized options for deployment of SFRs to transmute TRUs produced from all units of PWRs and PHWRs, a set of comparative scenarios are proposed as shown in Table 2.

While scenarios 1 and 2 are for the direct disposal of SNF, the remaining six scenarios are proposed to compare the effectiveness of the possible options for recycling of SNF in connection with SFRs. It was also assumed that the metallic SNF discharged from SFRs will be recycled in the Pyro-SFR fuel cycle based upon past reference studies [12, 13, 31].

Korea Atomic Energy Research Institute (KAERI) has been developing pyroprocessing technology in connection with SFR with a view to reducing the volume and the radiotoxicity of HLW (i.e., SNF) and thus to reducing the land area needed for direct disposal of SNF in deep geological formations [4].

It is also noted that the time needed for cooling the SNF (i.e., at least five years for the SNF from PWR and $\mathrm{PHWR}$ ) prior to pyroprocessing has been fully covered in this study, since enough amount of sufficiently cooled-down SNF produced from twenty-five units of NPPs since 1978 is already available for pyroprocessing in Korea [31]. Furthermore, the Korean government plans to commercialize a pyroprocessing facility along with SFR fuel fabrication plant by 2025 and then to deploy the first unit of SFR after three years later by 2028 , while just about 8 months is reported to be needed for SFR fuel fabrication $[6,32]$. Accordingly, it can be said that the SFR fuel fabrication time has been already taken into consideration in this study.

The values of characteristic parameters of representative SFRs listed in Table 3 for transmutation of TRUs in effective ways were obtained from a few design studies on SFRs, which were used for calculations in this study $[33,34]$. The characteristic data for SFR-1 and SFR-2 are generally taken from [33] and [34], respectively; however, the thermal efficiency of SFR-2 is assumed to be the same as SFR-1 since the specific thermal efficiency of SFR-2 is not available in the literature review.

For the effective transmutation of TRUs, SFRs with CR $=0.46$ and 0.6 have been assumed to be coupled with pyroprocessing in order to transmute all transuranic inventory generation from NPPs. The impact of CR on the SFR was determined by the consumption of TRUs as it is reported that the SFR with higher CR burns less TRUs, while the SFR with lower CR transmutes more. Thus, the minimum number of SFRs to be deployed for transmutation of all the inventory of TRUs can be eventually decided by the value of CR of a specific SFR design [13, 33-35]. 
TABLE 1: Dates of commercial operation and initial and to-be-extended design lifetimes for thirty-six units of Korean NPPs [27].

\begin{tabular}{|c|c|c|c|c|c|}
\hline Number & Reactor & $\begin{array}{l}\text { Electric power } \\
\quad\left(\mathrm{MWe}^{*}\right)\end{array}$ & $\begin{array}{c}\text { Commercial } \\
\text { operation }\end{array}$ & $\begin{array}{c}\text { Date of } \\
\begin{array}{c}\text { End of initial design } \\
\text { lifetime }\end{array} \\
\end{array}$ & $\begin{array}{c}\text { End of extended design lifetime } \\
\text { assumed }\end{array}$ \\
\hline 1 & Kori-1 & 587 & 1978-04-29 & $2017-06-19^{*}$ & No further extension ${ }^{* *}$ \\
\hline 2 & Kori-2 & 650 & $1983-07-25$ & $2023-07-25$ & $2043-07-25$ \\
\hline 3 & Kori-3 & 950 & 1985-09-30 & 2025-09-30 & 2045-09-30 \\
\hline 4 & Kori-4 & 950 & 1986-04-29 & 2026-04-29 & 2046-04-29 \\
\hline 5 & Shin Kori-1 & 1000 & 2011-02-28 & $2051-02-28$ & 2071-02-28 \\
\hline 6 & Shin Kori-2 & 1000 & $2012-07-20$ & $2052-07-20$ & 2072-07-20 \\
\hline 7 & Shin Wolsong-1 & 1000 & $2012-07-31$ & $2052-07-31$ & $2072-07-31$ \\
\hline 8 & Shin Wolsong-2 & 1000 & 2015-07-24 & 2055-07-24 & 2075-07-24 \\
\hline 9 & Hanul-1 & 950 & $1988-09-10$ & 2028-09-10 & 2048-09-10 \\
\hline 10 & Hanul-2 & 950 & 1989-09-30 & 2029-09-30 & 2049-09-30 \\
\hline 11 & Hanul-3 & 1000 & 1998-08-11 & 2038-08-11 & 2058-08-11 \\
\hline 12 & Hanul-4 & 1000 & $1999-12-03$ & $2039-12-03$ & $2059-12-03$ \\
\hline 13 & Hanul-5 & 1000 & $2004-12-31$ & $2044-12-31$ & $2064-12-31$ \\
\hline 14 & Hanul-6 & 1000 & 2005-04-22 & $2045-04-22$ & $2065-04-22$ \\
\hline 15 & Hanbit-1 & 950 & 1986-08-25 & $2026-08-25$ & $2046-08-25$ \\
\hline 16 & Hanbit-2 & 950 & $1987-06-10$ & 2027-06-10 & $2047-06-10$ \\
\hline 17 & Hanbit-3 & 1000 & 1995-03-31 & $2035-03-31$ & $2055-03-31$ \\
\hline 18 & Hanbit-4 & 1000 & 1996-01-01 & 2036-01-01 & 2056-01-01 \\
\hline 19 & Hanbit-5 & 1000 & $2002-05-21$ & $2042-05-21$ & $2062-05-21$ \\
\hline 20 & Hanbit-6 & 1000 & $2002-12-24$ & 2042-12-24 & 2062-12-24 \\
\hline 21 & Wolsong-1 & 679 & $1983-04-22$ & $2022-01-01^{* * *}$ & $2033-04-22^{* *}$ \\
\hline 22 & Wolsong-2 & 700 & 1997-07-01 & 2027-07-01 & 2047-07-01 \\
\hline 23 & Wolsong-3 & 700 & 1998-07-01 & 2028-07-01 & 2048-07-01 \\
\hline 24 & Wolsong-4 & 700 & 1999-10-01 & 2029-10-01 & 2049-10-01 \\
\hline 25 & Shin Kori-3 & 1400 & 2016-04-01 & 2076-04-01 & 2096-04-01 \\
\hline 26 & Shin Kori-4 & 1400 & 2017-02-01 & 2077-02-01 & 2097-02-01 \\
\hline 27 & Shin Kori-5 & 1400 & 2021-03-01 & 2081-03-01 & 2101-03-01 \\
\hline 28 & Shin Kori-6 & 1400 & 2022-03-01 & 2082-03-01 & 2102-03-01 \\
\hline 29 & Shin Hanul-1 & 1400 & 2017-04-01 & 2077-04-01 & 2097-04-01 \\
\hline 30 & Shin Hanul-2 & 1400 & 2018-02-01 & 2078-02-01 & 2098-02-01 \\
\hline 31 & Shin Hanul-3 & 1400 & $2022-12-01$ & $2082-12-01$ & $2102-12-01$ \\
\hline 32 & Shin Hanul-4 & 1400 & $2023-12-01$ & $2083-12-01$ & $2103-12-01$ \\
\hline 33 & Cheonji-1 & 1500 & $2026-12-01$ & 2086-12-01 & $2106-12-01$ \\
\hline 34 & Cheonji-2 & 1500 & $2027-12-01$ & $2087-12-01$ & $2107-12-01$ \\
\hline 35 & Shin Kori-7 & 1500 & $2028-12-01$ & $2088-12-01$ & $2108-12-01$ \\
\hline 36 & Shin Kori-8 & 1500 & $2029-12-01$ & 2089-12-01 & $2109-12-01$ \\
\hline
\end{tabular}

${ }^{*}$ MWe: megawatt electric. ${ }^{* *}$ Kori Unit 1 is assumed to be shut down on June 19, 2017, permanently. ${ }^{* * *}$ Second continued operation of 10 years is assumed for Wolsong Unit 1.

2.4. Fuel Cycles Cost Estimation. For the sake of nuclear fuel cycle cost estimation, both equilibrium and dynamic models are applicable to assess the economic feasibilities of the different fuel cycle options. The main difference between the two models is that the latter can be used for long-term cost calculation as time elapses while the former is based on the cost calculation at a certain base year $[12,14]$. The equilibrium model is capable of providing cost information for prompt decision-making by evaluating the worthiness of a nuclear fuel cycle option quantitatively [12, 14]. Therefore, the equilibrium model was chosen in this study to generate a series of cost information that may be helpful to understand the economic priorities of diverse nuclear fuel cycle options comprehensively and analytically.

To evaluate the economic feasibility of a nuclear fuel cycle through equilibrium model, Equations (i) to (xiv) shown in 
TABLE 2: Assumed strategic scenarios for transmutation of TRUs in SNF.

\begin{tabular}{|c|c|c|c|c|c|}
\hline \multirow{2}{*}{ Case } & \multirow{2}{*}{ Scenario } & \multirow{2}{*}{ Direct disposal/recycle } & \multicolumn{2}{|c|}{ Design lifetime } & \multirow{2}{*}{ SFR's CR } \\
\hline & & & NPPs (PWRs and PHWRs) & SFR & \\
\hline \multirow{2}{*}{ I } & SC-1 & Direct disposal & Design lifetime & N/A & N/A \\
\hline & $\mathrm{SC}-2$ & Direct disposal & $+20 y$ & N/A & N/A \\
\hline \multirow{2}{*}{ II } & SC-3 & Recycle & Design lifetime & Design lifetime & 0.46 \\
\hline & $\mathrm{SC}-4$ & Recycle & Design lifetime & Design lifetime & 0.6 \\
\hline \multirow{2}{*}{ III } & SC-5 & Recycle & $+20 y$ & Design lifetime & 0.46 \\
\hline & SC-6 & Recycle & $+20 y$ & Design lifetime & 0.6 \\
\hline \multirow{2}{*}{ IV } & SC-7 & Recycle & $+20 y$ & $+20 y$ & 0.46 \\
\hline & SC- 8 & Recycle & $+20 y$ & $+20 y$ & 0.6 \\
\hline
\end{tabular}

SC: scenario; CR: conversion ratio; N/A: not applicable.

TABLE 3: Characteristics of the reference SFRs.

\begin{tabular}{|c|c|c|c|c|}
\hline Reactor design & SFR-1 & & SFR-2 & Unit \\
\hline Electric power & & 600 & & MWe \\
\hline Thermal efficiency & & 39.38 & & $\%$ \\
\hline Thermal power & & 1523.4 & & MWth \\
\hline Capacity factor & & 85 & & $\%$ \\
\hline Cycle length & & 332 & & EFPD \\
\hline Design lifetime (normal) & & 60 & & Years \\
\hline Design lifetime (extended) & & 80 & & Years \\
\hline Conversion ratio & 0.46 & & 0.6 & - \\
\hline Number of batches & 6 & & 4 & - \\
\hline Discharge burnup & 217 & & 116.3 & GWD/MTHM \\
\hline TRU consumption rate & 530 & & 307.1 & $\mathrm{~kg} /$ cycle \\
\hline Fuel type & TRU-W-10Zr metal & & TRU-U-10Zr metal & Wt.\% \\
\hline Annual electricity generation & 4778.62 & & 4770.41 & GWh \\
\hline
\end{tabular}

MWth: megawatt thermal; EFPD: effective full-power day; GWD: gigawatt days; MTHM: metric ton heavy metal; Kg: kilogram; Wt.\%: weight percent; GWh: gigawatt hour.

Table 12 were proposed to calculate the fuel cycle costs with an assumption of zero discount rate [12]. All the unit cost data are discounted to the base year of 2016 with an inflation rate of $2.08 \%$. In order to calculate the fuel cycle cost (FCC) for each fuel cycle, the individual process cost (i.e., the unit cost multiplied by the material quantity at each step of a fuel cycle) should be calculated. In case of PWR, Equations (i) to (iii) and (vi) can be used to calculate the individual process cost. The total process cost of nuclear fuel cycle can be defined as a summation of all the individual process costs involved in the respective fuel cycle and calculated as such. In case of PWR, PHWR, and Pyro-SFR fuel cycles, the total process cost of nuclear fuel cycle can be calculated by use of Equations (viii) to (x), respectively. The FCC can be defined as the total process cost for a fuel cycle normalized to the electricity generation per unit mass and calculated by dividing the total process cost for each fuel cycle with electricity generation per unit mass. Equations (xi) to (xiv) are used to calculate the FCCs of PWR, PHWR, and Pyro-SFR fuel cycles, respectively. Calculation method and equations are referred from past studies on the equilibrium mass flow and cost model in nuclear fuel cycle $[12,36,37]$.
In this study, however, Equations (xi) and (xii) were modified in terms of general design parameters of PWRs and PHWRs, while Equations (xiii) and (xiv) were modified based on the specific design parameters of Korean SFRs [33, 34]. Equations (ii) to (vi) were modified by including the cost for disposal of process waste being generated from processes of each fuel cycle [37]. Annual fuel requirement $\left(A_{\mathrm{FR}}\right)$ in MTHM and annual electricity generation $\left(A_{\mathrm{EG}}\right)$ in GWh from NPPs (i.e., PWRs and PHWRs) and SFRs are estimated by

$$
\begin{aligned}
& A_{\mathrm{FR}}=\frac{P \times \mathrm{FCL}}{\varepsilon \times \mathrm{BU}} \\
& A_{\mathrm{EG}}=A_{\mathrm{FR}} \times\left(\frac{\mathrm{BU}}{1,000}\right) \times \varepsilon \times\left(\frac{24 \mathrm{~h}}{1 \mathrm{~d}}\right),
\end{aligned}
$$

where $P$ is the electric power of a reactor (MWe), FCL is the length of a cycle (EFPD), $\varepsilon$ is the thermal efficiency, and BU is the discharge burnup (MWD/MTHM). It is noted that the annual electricity generation for SFR-1 and SFR-2 in Table 3 can be also calculated by using (4). 


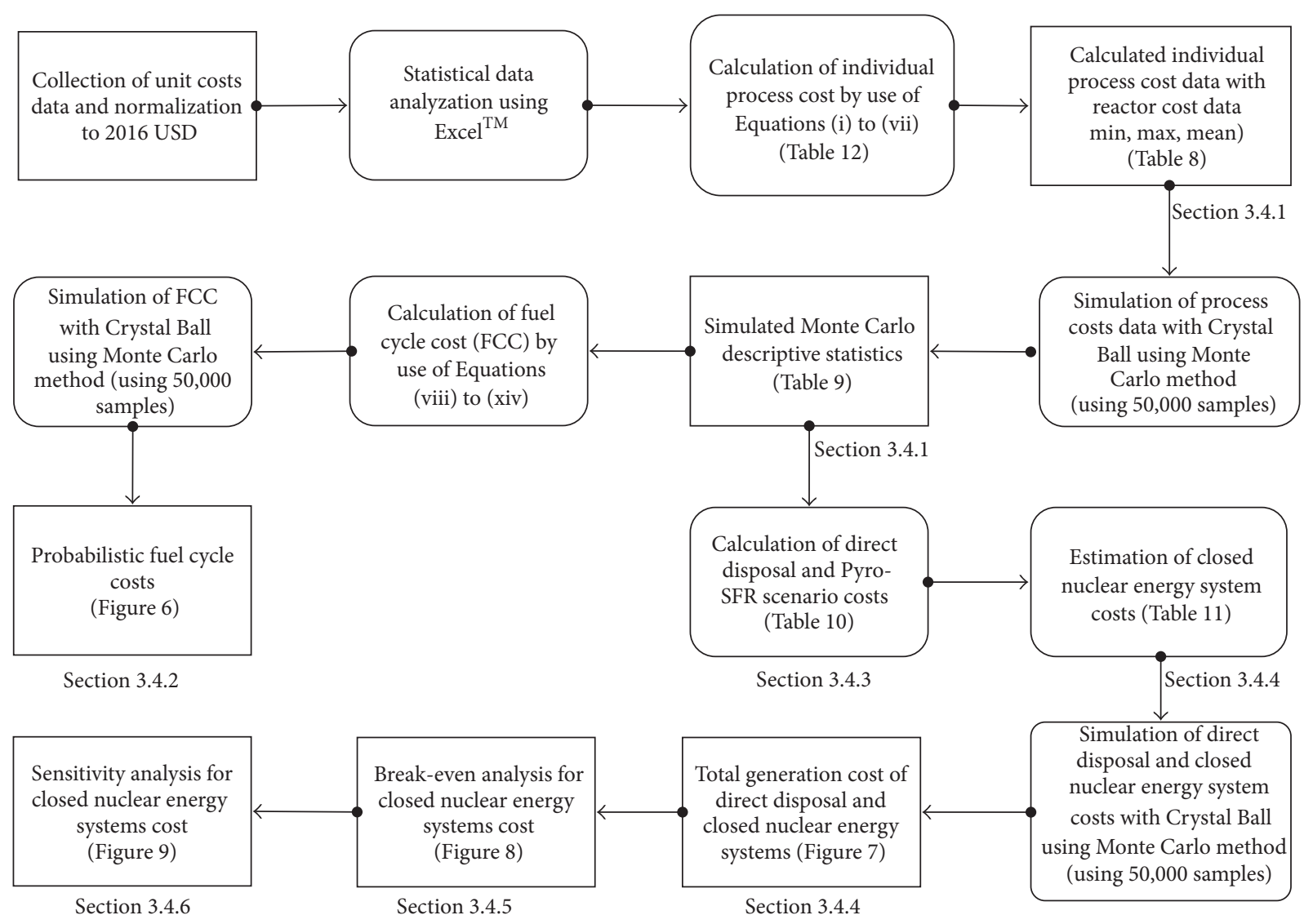

FIGURE 2: Overall procedure to estimate cost for economic analysis adopted in this study.

The whole process of economic analysis established and conducted in this study is schematically summarized as shown in Figure 2.

\section{Results and Discussion}

3.1. Estimation of Total Inventory of SNF and TRUs. As shown in Table 4, the total amount of SNF generated from twentyfour units of operating NPPs as of March 2016 was calculated to be 14,511 metric tons of heavy metal (MTHM) by the NFCSS and the information given in Table 1 . The result is quite comparable to the officially reported total inventory of SNF in terms of mass (i.e., 14,608 MTU) accumulated as of March 2016 within the relative error of less than 0.7 percent [1].

In addition, the overall inventory of SNF to be produced from thirty-six units of NPPs during their initial design lifetimes only and additional continued operation periods (except for Kori Unit 1) were estimated to be 41,718 and 61,232 MTHM, respectively. That is, about 2.9 to 4.2 times more SNF in mass than the present inventory as of March 2016 will be accumulated if an additional eight units of APR1400 and four units of APR+ type PWRs are deployed by 2029 as officially planned. It is also noted that the potential of continued operation of NPPs up to an additional twenty years may affect the total national inventory of SNF by a factor of $1.47(\approx 61,232 / 41,718)$.
Table 5 shows the estimated mass (in grams) of each TRU nuclide which already exists in the total inventory of SNF generated from NPPs as of 2016 and the SNF to be further generated from all NPPs in operation and under construction or planning. It is noted that the mass of fission products is not included in Table 5, since this study mainly focuses on the radiotoxicity of the SNF in the long term as addressed in Section 2.2.

The continued operation of NPPs for up to an additional twenty years may increase the mass of each TRU nuclide by $41 \%$ in average. The increment of total inventory of TRUs produced from operation of NPPs for their design lifetimes or for longer continued operation turns out to be about $385 \%$ or $583 \%$ in average compared to the inventory as of March 2016.

However, there might be a few reasons for the partially higher increment of specific TRUs such as ${ }^{243} \mathrm{Am}$ and ${ }^{244} \mathrm{Cm}$. In order to find the reason of the partially higher increase of ${ }^{243} \mathrm{Am}$ and ${ }^{244} \mathrm{Cm}$, the fraction of each TRU nuclide from different types of Korean NPPs (e.g., APR-1400, $1000 \mathrm{MWe}$ PWR, and PHWR) was calculated and compared. As a result, it turns out that the fractions of ${ }^{243} \mathrm{Am}$ and ${ }^{244} \mathrm{Cm}$ from APR1400 and APR+ NPPs are 2.2 and 3.75 times higher than those from 1000 MWe PWR as shown in Table 6. Secondly, specifically in case of ${ }^{243} \mathrm{Am}$, the higher increment may be ascribed to the higher burnup of $60 \mathrm{GWD} / \mathrm{MTHM}$ for APR1400 and APR+, since the production of ${ }^{243} \mathrm{Am}$ increases exponentially with burnup [38]. 
TABLE 4: Total amount of SNF to be generated from operation of all units of PWRs and PHWRs planned to be deployed by 2029 per reactor site.

\section{Amount of SNF (MTHM)}

Site As of March 2016

With operation for initial design lifetime $(2089)^{*}$
With continued operation for an additional 20 years

$(2109)^{*}$

\begin{tabular}{lcccc}
\multicolumn{1}{c}{ This study } & {$[1]$ (MTU) } & & \\
\hline Kori (PWRs) & 2,201 & 2,294 & 11,905 & 16,282 \\
Hanul (PWRs) & 2,049 & 2,043 & 9,716 & 13,697 \\
Hanbit (PWRs) & 2,391 & 2,403 & 4,502 & 6,753 \\
Shin-Wolsong (PWRs) & 65 & 54 & 1,203 & 1,804 \\
Wolsong (PHWRs) & 7,805 & 7,815 & 11,514 & 18,858 \\
Cheonji (PWRs) & - & - & 2,878 & 3,837 \\
Total & 14,511 & 14,608 & 41,718 & 61,232 \\
\hline
\end{tabular}

${ }^{*}$ Results for the year of 2089 represent the scenario where continued operation of 36 units of NPPs is not assumed, and those for the year of 2109 are for the scenario where continued operation of all NPPs except for Kori Unit 1 is taken into account. In addition, the contribution of fission products in the spent nuclear fuel is not included in this table.

TABLE 5: Total mass of TRUs to be produced and accumulated from operation of all units of NPPs (PWRs, PHWRs) planned to be deployed by $2029^{*}$.

\begin{tabular}{|c|c|c|c|c|c|c|c|}
\hline \multirow{2}{*}{ Nuclide } & \multirow{2}{*}{ Half-life (y) } & \multicolumn{3}{|c|}{ Total mass of TRUs (g) as of } & \multicolumn{3}{|c|}{ Percentage of added mass for the period (\%) } \\
\hline & & 2016 & 2089 & 2109 & 2016 to 2089 & 2016 to 2109 & 2089 to 2109 \\
\hline${ }^{241} \mathrm{Am}$ & $4.32 E+02$ & $3.79 E+05$ & $1.70 E+06$ & $2.39 E+06$ & $349 \%$ & $534 \%$ & $41 \%$ \\
\hline${ }^{242 \mathrm{~m}} \mathrm{Am}$ & $1.41 E+02$ & $8.54 E+03$ & $4.02 E+04$ & $5.65 E+04$ & $371 \%$ & $565 \%$ & $41 \%$ \\
\hline${ }^{243} \mathrm{Am}$ & $7.37 E+03$ & $1.31 E+06$ & $1.26 E+07$ & $1.72 E+07$ & $862 \%$ & $1220 \%$ & $37 \%$ \\
\hline${ }^{242} \mathrm{Cm}$ & $1.63 E+02$ & $1.48 E+05$ & $8.86 E+05$ & $1.23 E+06$ & $500 \%$ & $737 \%$ & $39 \%$ \\
\hline${ }^{244} \mathrm{Cm}$ & $1.81 E+01$ & $4.92 E+05$ & $7.90 E+06$ & $1.07 E+07$ & $1506 \%$ & $2080 \%$ & $36 \%$ \\
\hline${ }^{237} \mathrm{~Np}$ & $2.14 E+06$ & $5.73 E+06$ & $2.43 E+07$ & $3.43 E+07$ & $324 \%$ & $502 \%$ & $42 \%$ \\
\hline${ }^{238} \mathrm{Pu}$ & $8.77 E+01$ & $2.19 E+06$ & $1.24 E+07$ & $1.73 E+07$ & $466 \%$ & $692 \%$ & $40 \%$ \\
\hline${ }^{239} \mathrm{Pu}$ & $2.41 E+04$ & $5.52 E+07$ & $1.84 E+08$ & $2.65 E+08$ & $233 \%$ & $383 \%$ & $45 \%$ \\
\hline${ }^{240} \mathrm{Pu}$ & $6.56 E+03$ & $2.61 E+07$ & $9.59 E+07$ & $1.37 E+08$ & $267 \%$ & $428 \%$ & $44 \%$ \\
\hline${ }^{241} \mathrm{Pu}$ & $1.43 E+01$ & $1.22 E+07$ & $5.47 E+07$ & $7.70 E+07$ & $348 \%$ & $534 \%$ & $42 \%$ \\
\hline${ }^{242} \mathrm{Pu}$ & $3.73 E+05$ & $5.53 E+06$ & $3.62 E+07$ & $5.01 E+07$ & $553 \%$ & $809 \%$ & $39 \%$ \\
\hline Total & & $1.09 E+08$ & $4.30 E+08$ & $6.16 E+08$ & $385 \%$ & $583 \%$ & $41 \%$ \\
\hline
\end{tabular}

${ }^{*}$ Results for the year of 2089 represent the scenario where continued operation of 36 units of NPPs is not assumed, and those for the year of 2109 are for the scenario where continued operation of all NPPs except for Kori Unit 1 is taken into account.

TABLE 6: Fractions of ${ }^{243} \mathrm{Am}$ and ${ }^{244} \mathrm{Cm}$ generated from different types of Korean NPPs.

\begin{tabular}{lcc}
\hline Reactor & \multicolumn{2}{c}{ Radionuclide } \\
& ${ }^{243} \mathrm{Am}$ & ${ }^{244} \mathrm{Cm}$ \\
\hline PHWR & $4.066 E-04$ & $3.742 E-05$ \\
$1000 \mathrm{MWe}$ PWR & $1.977 E-02$ & $8.169 E-03$ \\
APR-1400 & $4.371 E-02$ & $3.068 E-02$ \\
APR+ & $4.371 E-02$ & $3.068 E-02$ \\
\hline
\end{tabular}

3.2. Estimation of Nominal Radiological Risks. Figure 3 shows the time-dependent nominal radiotoxicity index (in Sv/g) for the total inventory of TRUs in SNF to be generated from all thirty-six units of NPPs without continued operation, which was calculated by (2) and (3) using the values of parameters in Table 5. Figure 3 also shows the general trend of timedependent decreasing of radiotoxicity index and dominant TRU nuclides along with elapsed time from the year of 2089, in which the last NPP's initial lifetime will be ended. During the first sixteen years, the total radiotoxicity index is controlled by ${ }^{244} \mathrm{Cm}$, and then it is controlled by ${ }^{238} \mathrm{Pu}$ until 250 years from its initial decay. Subsequently, the most important TRU nuclides are ${ }^{240} \mathrm{Pu}$ until 8,200 years and ${ }^{239} \mathrm{Pu}$ until 260,000 years, and then ${ }^{242} \mathrm{Pu}$ and ${ }^{237} \mathrm{~Np}$ dominate the radiotoxicity index subsequently for relative comparison.

The relative ingestion radiotoxicity index of natural uranium is also plotted. The ingestion radiotoxicity index of the natural uranium was calculated from the specific activity $(25,280 \mathrm{~Bq} / \mathrm{g})$ and ingestion dose coefficient for adult ( $31.7 \mathrm{mSv} / \mathrm{g}$ ) of natural uranium [39]. It can be said that the radiotoxicity index of TRUs in total inventory of SNF will decrease down to the level of the natural uranium only after 


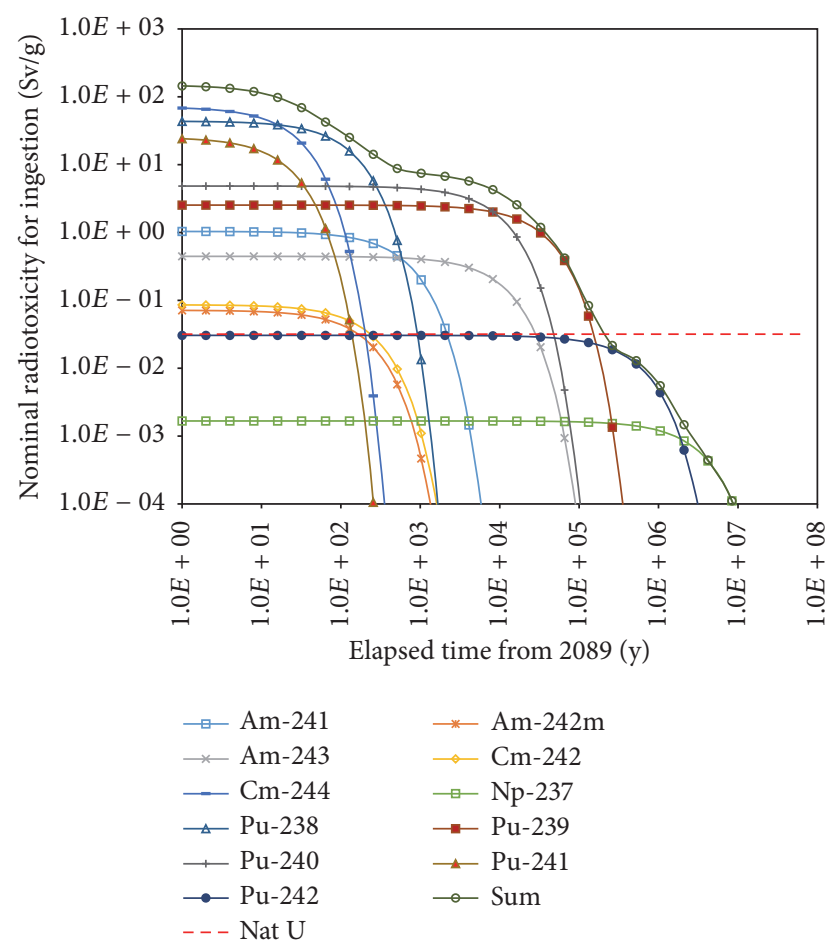

FIGURE 3: Nominal radiotoxicity for ingestion of TRUs in SNF from NPPs in comparison with natural uranium along with elapsed time from 2089 without consideration of the possibility of continued operation.

around 200,000-year-long radioactive decay. The shape of curves and time-dependent decreasing trend of the sum of the total radiotoxicity indices of TRUs in Figure 3 conform to those reported in other studies including textbooks dealing with the toxicity of SNF discharged from nuclear reactors, which demonstrates the feasibility of the calculation conducted and the results obtained in this study [29, 30].

\subsection{Establishment of Strategic Scenarios to Transmute TRUs} by SFRs. It was assumed that all TRUs present in SNF from all units of NPPs are to be transmuted in the SFRs by utilizing Pyro-SFR fuel cycle scenarios. In order to formulate the quantified transmutation schemes for TRUs with the incorporation of Pyro-SFR, basic scenarios in Table 2 have been expanded as shown in Table 7, which shows the minimum number of SFRs to be required for each scenario along with the duration of commercialization of SFRs. As described above, the deployment of APR-1400 and APR+ by 2029 and SFRs from 2028 to their design or extended lifetime was assumed based upon the official national plans promulgated by the Korean government $[2,4]$. However, additional NPPs (PWRs and PHWRs) and/or SFRs may be deployed thereafter beyond the time frame covered in the above national plans, which was not taken into account in this study because long-term forecast without sound basis may cause very high uncertainties and is not compatible with the main objective of this study.

Deployment rate of SFRs directly affects the elements of modeled transmutation scenarios such as the number of
SFRs needed, the transmutation rate, and the target year of full transmutation of TRUs. In the case of PWRs and PHWRs, the deployment rate in average was calculated from the historical rate of commercialization of NPPs in Korea and the government's future deployment plan for new reactors as announced. Totally, thirty-six units of NPPs have been and will be deployed from the first commercial operation of Kori Unit 1 in 1978 till the commercialization of the thirty-sixth reactor in 2029. That is, the average deployment rate of NPPs in Korea is 1.44 years (i.e., 17 months) per reactor. In case of SFR deployment in scenarios SC-3 to SC-8, however, it was assumed that each unit of SFR will be deployed every 1.67 years (i.e., 20 months) after the commercialization of the first SFR in 2028 in order to transmute all the inventory of TRUs generated from the operation of all thirty-six NPPs to be installed by 2029. As the SFR will be the first-of-akind (FOAK) reactor to be deployed in Korea, the average deployment rate of SFR was proposed to be $15 \%$ longer than the deployment rate of NPPs (i.e., PWRs and PHWRs) in this study.

It is shown that at least fourteen to thirty-four units of SFRs should be used for full transmutation of TRUs from thirty-six units of NPPs, which mainly depends upon the design parameters (e.g., CR) of SFR, life extension of nuclear reactors, and so forth. It is noted that the SFR design with lower CR (0.46) such as in scenarios SC-3, SC-5, and SC7 requires a smaller number of SFRs compared to scenarios SC-4, SC-6, and SC-8 which burn less TRUs per cycle due to higher CR (0.6). Therefore, more units of SFRs are needed and thus longer time duration of transmutation is required for scenarios SC-4, SC-6, and SC-8 than SC-3, SC-5, and SC-7 as shown in Table 7.

Some investigators have reported different numbers of SFRs (i.e., minimum of 29 to 45 or more) to be deployed in Korea to transmute TRUs based upon their own assumptions, in which two types of SFRs (i.e., break-even reactors with CR of no more than 1 and burners with $\mathrm{CR}$ of 0.61 to 0.70 ) were assumed $[8,13,18]$. In this study, we have considered two types of SFRs as burners (CR of 0.46 and 0.6 ) as one of the primary objectives of this study is to simulate the full-scale transmutation of TRUs by deploying SFRs with a minimum of 21-year and maximum of 55-year-long strategic planning as shown in Table 7.

Figures 4 and 5 show that TRUs generated from scenario SC-1 (i.e., operation of NPPs for design lifetimes) can be fully transmuted by the years of 2109 to 2126 with SC-3 and SC-4, respectively, and TRUs from SC-2 (i.e., extended operation of NPPs up to twenty years) by the years of 2119, 2143, 2133, and 2148 with SC-5, SC-6, SC-7, and SC-8, respectively, by the use of the minimum number of SFRs as shown in Table 7.

The minimum number of SFRs needed for each scenario in Table 7 was calculated by the use of the following equation:

$$
N_{\mathrm{SFR}}=\frac{A_{\mathrm{TRU}-\mathrm{NFCSS}}}{C_{\mathrm{TRU}} \times \mathrm{OPR}_{t}},
$$

where $A_{\text {TRU-NFCSS }}$ is the amount of TRUs estimated by NFCSS (MTHM), $C_{\text {TRU }}$ is the consumption rate of TRUs per reactoryear (MTHM/reactor.y), and $\mathrm{OPR}_{t}$ is the operational period of a reactor $(y)$. 
TABLE 7: Strategic scenarios for transmutation of TRUs.

\begin{tabular}{lcc}
\hline Case I & Scenario SC-1 & Scenario SC-2 \\
\hline Number of PWRs & 32 & 32 \\
Number of PHWRs & 4 & 4 \\
Deployment period of PWRs and PHWRs & $1978-2029$ (51 years) & $1978-2029(51$ years) \\
MAs/Pu generated (MT) & $47.38 / 383.08$ & $66.14 / 549.43$ \\
\hline Case II & Scenario SC-3 & Scenario SC-4 \\
\hline MAs/TRUs generated in SC-1 & $47.38 / 430.47$ & $47.38 / 430.47$ \\
Minimum number of SFRs needed & 14 & 24 \\
Deployment period of SFRs & $2028-2049$ (21 years) \\
Case III & Scenario SC-5 & Scenario SC-6 \\
\hline MAs/TRUs generated in SC-2 & $66.14 / 615.57$ & $66.14 / 615.57$ \\
Minimum number of SFRs needed & 20 & 34 \\
Deployment period of SFRs & $2028-2059$ (31 years) \\
Case IV & Scenario SC-7 & $2028-2066(38$ years) \\
\hline MAs/TRUs generated in SC-2 & $66.14 / 615.57$ & Scenario SC-8 \\
Minimum number of SFRs needed & 15 & $66.14 / 615.57$ \\
Deployment period of SFRs & $2028-2051$ (23 years) & 25 \\
\hline
\end{tabular}

MT: metric ton; MAs: minor actinides; Pu: plutonium.

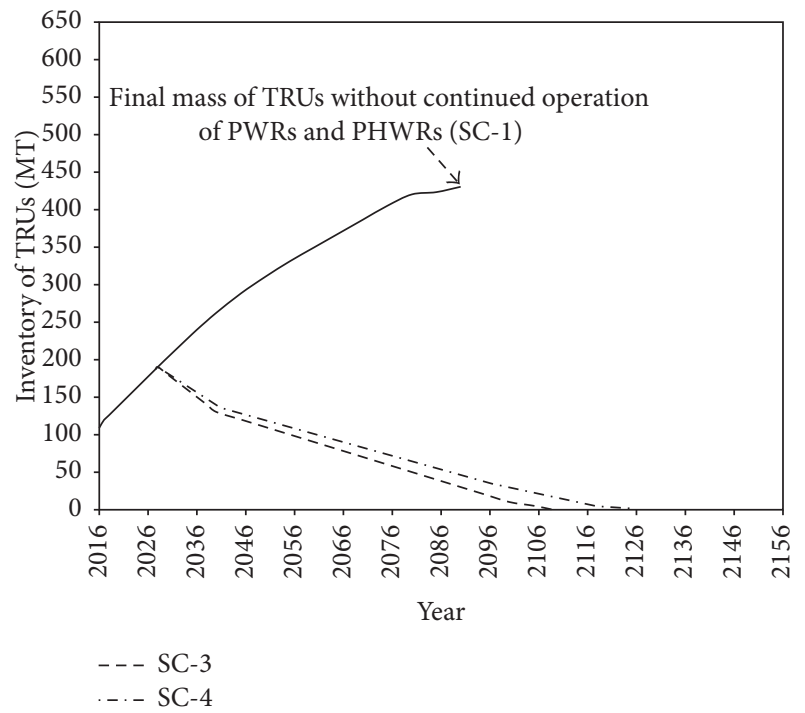

FIGURE 4: Prospective reduction of mass of TRUs inventory depending upon two different scenarios in which PWRs and PHWRs are operated for design lifetimes.

As noticed, the scope of this study is to simulate the deployment of NPPs (i.e., PWRs and PHWRs) by the year of 2029 and SFRs from the year of 2028 until their operational or extended lifetime. Further deployment of NPPs and SFRs is not taken into account based on the 7th Basic Plan on Electricity Demand and Supply which includes the latest national policy and strategies to construct an additional eight units of APR-1400 and four units of APR+ by 2029 only. There is no specific plan to deploy NPPs after 2100 [2]. Since the first feed of nuclear fuel for SFRs will be manufactured from TRUs in the SNF produced from NPPs (i.e., PWRs and PHWRs), deployment of additional SFRs would not be assumed when

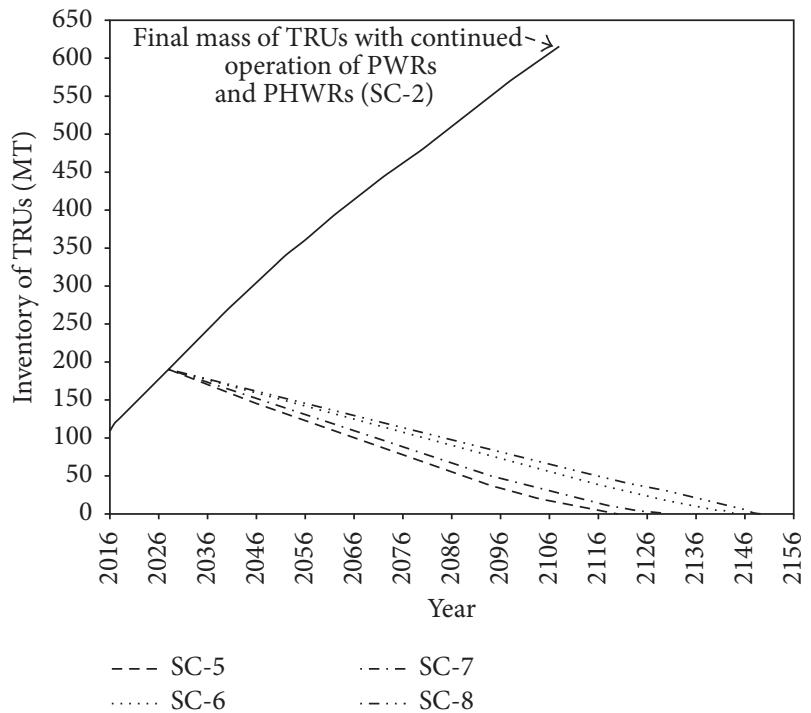

FIGURE 5: Prospective reduction of mass of TRUs inventory depending upon four different scenarios in which life extension of PWRs, PHWRs, and SFRs is allowed.

the NPPs (i.e., origin of the nuclear fuel for SFR) are not to be present in the future $[13,31]$. Moreover, the basic methodology proposed in this study would be applicable if the government plans to deploy new nuclear reactors in the future.

\subsection{Estimation of Cost for Direct Disposal and Pyro-SFR Fuel Cycle Options}

3.4.1. Derivation of Process Costs Based upon Unit Costs from Existing Studies. In order to perform front- and back-end fuel cycle cost calculations, a variety of economic studies on nuclear fuel cycles were firstly reviewed and analyzed. 
TABLE 8: Probability distribution function of nuclear fuel cycle process costs and reactor cost (based on 2016 USD).

\begin{tabular}{|c|c|c|c|c|c|c|}
\hline Category & Probability distribution & Min & $\operatorname{Max}$ & Mean & Unit & References \\
\hline Uranium $^{*}$ & Triangular [37] & 58 & 409 & 219 & $\$ / \mathrm{kgU}$ & {$[14-18,21,35-37,40-44]$} \\
\hline Conversion & Uniform [12] & 6 & 33 & 14 & $\$ / \mathrm{kgU}$ & {$[14-18,21,35-37,40-44]$} \\
\hline Enrichment & Uniform [12] & 262 & 411 & 481 & \$/SWU & {$[14-18,21,35-37,40-44]$} \\
\hline PWR fuel fabrication & Triangular [37] & 281 & 422 & 346 & $\$ / \mathrm{kgHM}$ & {$[14-18,21,35-37,40-44]$} \\
\hline PHWR fuel fabrication & Triangular [37] & 60 & 186 & 152 & $\$ / \mathrm{kgHM}$ & {$[17-21,35,36,43]$} \\
\hline $\mathrm{UO}_{2}$-pyroprocess & Triangular [37] & 882 & 4,819 & 3,064 & $\$ / \mathrm{kgHM}$ & {$[14-16,18-20,35,36]$} \\
\hline SFR-pyroprocess & Triangular [37] & 1,392 & 5,692 & 3,786 & $\$ / \mathrm{kgHM}$ & {$[14-16,18-20,35,36]$} \\
\hline SFR fuel fabrication & Triangular [37] & 2,297 & 8,680 & 4,939 & $\$ / \mathrm{kgHM}$ & {$[14-16,18-20,35-37,40,42]$} \\
\hline PWR IS\&T & Triangular [37] & 133 & 925 & 480 & $\$ / \mathrm{kgHM}$ & {$[14-21,35-37,40-43]$} \\
\hline PHWR IS\&T & Triangular [37] & 64 & 701 & 393 & $\$ / \mathrm{kgHM}$ & {$[17-21]$} \\
\hline Pyroprocess HLW (storage) ${ }^{*}$ & Triangular [37] & 124,561 & 231,612 & 199,692 & $\$ / \mathrm{m}^{3}$ & {$[15,16,19,20]$} \\
\hline LILW-SL disposal ${ }^{*}$ & Triangular [37] & 3,465 & 4,170 & 3,818 & $\$ / \mathrm{m}^{3}$ & {$[15,16,37,40]$} \\
\hline LILW-LL disposal ${ }^{*}$ & Triangular [37] & 9,240 & 11,121 & 9,867 & $\$ / \mathrm{m}^{3}$ & {$[15,16,37,40]$} \\
\hline PWR final disposal ${ }^{*}$ & Triangular [37] & 521 & 2,245 & 1,107 & $\$ / \mathrm{kgHM}$ & {$[14,17-21,37,40-43]$} \\
\hline PHWR final disposal* & Triangular [37] & 106 & 699 & 519 & $\$ / \mathrm{kgHM}$ & {$[17-21]$} \\
\hline Pyroprocess disposal ${ }^{*}$ & Triangular [37] & 17,361 & 493,893 & 296,539 & $\$ / \mathrm{kgHM}$ & {$[14,15,18-20,37,40,41,43]$} \\
\hline PWR overnight ${ }^{*}$ & Triangular [37] & 1,919 & 7,018 & 4,746 & $\$ / \mathrm{kWe}$ & {$[17,18,37,43]$} \\
\hline PHWR overnight* & Triangular [37] & 1,903 & 3,268 & 2,587 & $\$ / \mathrm{kWe}$ & {$[37]$} \\
\hline SFR overnight* & Triangular [37] & 2,331 & 9,199 & 6,115 & $\$ / \mathrm{kWe}$ & {$[17,18,40,43]$} \\
\hline
\end{tabular}

IS\&T: interim storage and transportation; HLW: high-level waste; LILW-SL: low intermediate level waste-short-lived; LILW-LL: low intermediate level wastelong-lived; Min: minimum; Max: maximum; kgU: kilogram uranium; SWU: separative work unit; KgHM: kilogram heavy metal; $\mathrm{m}^{3}$ : cubic meter; USD: US dollar. ${ }^{*}$ Values are adopted from different sources, normalized to 2016 USD, statistically analyzed, and presented in this table.

It is intended in this study to consider the variabilities and uncertainties of unit cost data as much as possible by expanding the references of unit cost data and using a statistical manipulation. As a result, various domestic and overseas unit cost data available from reliable information resources such as official reports of competent international organizations and selected scientific papers published in international journals were collected as shown in Table 8 [14-21, 35-37, 4044]. All the unit cost data were normalized to the 2016 US dollar values.

In this study, first unit cost data is collected from different studies and statistically analyzed, and then individual process costs (as shown in Table 8) were calculated by using the result of analysis as an input to (i) to (vii) from Table 12. The individual process costs were derived again by probabilistic calculation using a commercially available risk analysis software program called Crystal Ball ${ }^{\mathrm{TM}}$ utilizing Monte Carlo simulation, which randomly selects values for each input to the model from a distribution specified by the user. The calculation was done by use of (i) to (vii) from Table 12 after defining the parameters of probability distribution for the individual process costs of different nuclear fuel cycles. The other costs except for the individual process costs in Table 8 were derived from statistical analysis of reference unit costs. In accordance with the above methodology, the statistics of costs were calculated as shown in Table 9.

Precision of the cost values can be analyzed by using the data of coefficient of variability (CV) given in Table 9, which compares the variability of cost values relative to the mean or in other words precision of cost values. It is reportedly known that a parameter having a higher value of CV (i.e., dispersion around the mean is higher) is considered to be less precise. The values derived from Crystal Ball simulation can be said to be acceptable, since all values of CV in Table 9 range from 0 to 1 which are typically known to be precise enough [45].

Three types of probability distributions, uniform, triangular, and normal distributions, which are typically used for cases where limited amount of sample data is available, as shown in Table 8, were used for each cost element in order to quantify the uncertainties in estimation of the costs. The probability distribution of each cost in Table 8 was adopted from studies on economic analysis of nuclear fuel cycle [12, 37].

3.4.2. Calculation of Fuel Cycle Costs. Figure 6 shows the results of probabilistic calculation of the fuel cycle costs (FCCs) for selected fuel cycle options. With the assumption of probability distribution for each process cost element as stated in Table 8, a series of Monte Carlo simulations using Crystal Ball software were carried out for 50,000 samples to address the inherent uncertainties of the estimated cost. As a result of the simulation, the mean values of FCCs for PWR (direct disposal), PHWR (direct disposal), Pyro-SFR$1(\mathrm{CR}=0.46)$, and Pyro-SFR-2 $(\mathrm{CR}=0.6)$ were calculated basically by using (viii) to (xiv) from Table 12 to be 8.174 mills/ kWh, 22.114 mills/kWh, 6.065 mills/kWh, and 11.310 mills/ $\mathrm{kWh}$, respectively. 
TABLE 9: Descriptive statistics of process cost and reactor cost using 50,000 random samples with Monte Carlo method.

\begin{tabular}{|c|c|c|c|c|c|c|}
\hline Category & Minimum & Maximum & Mean & Median & $\mathrm{SD}$ & $\mathrm{CV}$ \\
\hline Uranium & 58.16 & 407.27 & 228.89 & 226.88 & 71.46 & 0.31 \\
\hline Conversion & 6.20 & 33.59 & 19.92 & 19.97 & 7.91 & 0.40 \\
\hline Enrichment & 406.21 & 576.50 & 487.52 & 486.34 & 31.08 & 0.06 \\
\hline PWR fuel fabrication & 284.63 & 425.83 & 352.40 & 351.10 & 29.04 & 0.08 \\
\hline PHWR fuel fabrication & 63.77 & 188.97 & 134.76 & 137.84 & 26.48 & 0.20 \\
\hline $\mathrm{UO}_{2}$-pyroprocess & 990.05 & $5,147.95$ & $3,063.97$ & $3,062.27$ & 809.89 & 0.26 \\
\hline SFR-pyroprocess & $1,514.36$ & $5,963.69$ & $3,765.25$ & $3,774.47$ & 879.01 & 0.23 \\
\hline SFR fuel fabrication & $2,322.11$ & $8,682.88$ & $5,311.27$ & $5,225.73$ & $1,315.30$ & 0.25 \\
\hline PWR IS\&T & 135.35 & 923.51 & 513.05 & 505.19 & 161.88 & 0.32 \\
\hline PHWR IS\&T & 65.51 & 699.21 & 385.24 & 386.78 & 130.25 & 0.34 \\
\hline Pyroprocess HLW (storage) & $124,855.22$ & $231,538.87$ & $185,416.02$ & $188,057.06$ & $22,407.71$ & 0.12 \\
\hline LILW-SL disposal & $3,467.53$ & $4,166.87$ & $3,817.85$ & $3,817.21$ & 144.40 & 0.04 \\
\hline LILW-LL disposal & $9,246.08$ & $11,119.19$ & $10,074.75$ & $10,033.59$ & 391.76 & 0.04 \\
\hline PWR final disposal & 523.49 & $2,244.14$ & $1,292.74$ & $1,254.76$ & 359.15 & 0.28 \\
\hline PHWR final disposal & 296.62 & 698.04 & 503.64 & 507.00 & 83.10 & 0.16 \\
\hline Pyroprocess disposal & $17,870.38$ & $492,381.02$ & $269,486.28$ & $274,929.70$ & $97,445.86$ & 0.36 \\
\hline PWR overnight & $1,907.61$ & $7,027.80$ & $4,758.43$ & $4,869.87$ & $1,067.17$ & 0.22 \\
\hline PHWR overnight & 105.46 & $1,896.52$ & $1,197.88$ & $1,258.74$ & 393.91 & 0.33 \\
\hline SFR overnight & $2,321.49$ & $9,196.44$ & $6,107.57$ & $6,254.42$ & $1,438.34$ & 0.24 \\
\hline
\end{tabular}

SD: standard deviation; CV: coefficient of variability. ${ }^{*}$ All units are the same as in Table 8 for each item.

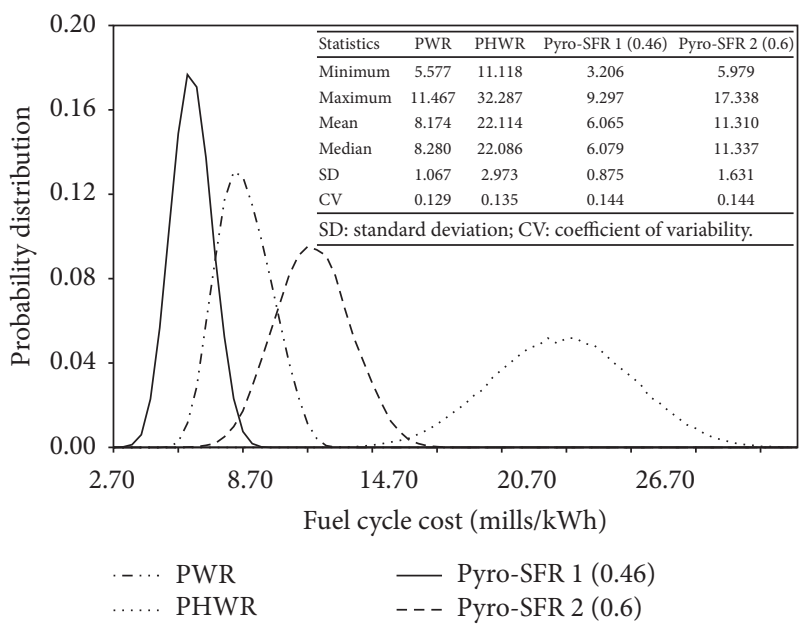

Figure 6: Probabilistic distribution of fuel cycle cost (FCC).

As shown in the statistics of Figure 6, the FCC for PyroSFR-1 $(\mathrm{CR}=0.46)$ is found to be the most economical fuel cycle option followed by PWR (direct disposal), Pyro-SFR-2 $(\mathrm{CR}=0.6)$, and PHWR (direct disposal). Difference between the FCCs of Pyro-SFR-1 and Pyro-SFR-2 mainly comes from the difference of the values of CR and burnups of SFRs between the two fuel cycles as shown in Table 3. As higher burnup generates more energy per unit mass of fuel; the mass of fuel required for SFR-2 is more than (almost double) that for SFR-1. Accordingly, the FCC in $\$ / \mathrm{kWh}$ for Pyro-SFR-1 turns out to be almost half that for Pyro-SFR-2 due to lower CR and higher burnup $[13,35,46]$. As can be seen from Figure 6, the FCC distribution of PHWR is wider than other fuel cycles and the distribution of Pyro-SFR-1 is narrower than others. It is noted that the peakedness and flatness of a distribution for PWR and Pyro-SFR-1 fuel cycles which resulted in Figure 6 are comparable with previous studies reported [14].

3.4.3. Calculation of Scenario Cost. The next step in economic analysis is to estimate the scenario-based cost. As discussed in Tables 2 and 7, four cases each consisting of two scenarios are analyzed and presented in Table 10, where the overall scenario cost including overnight cost, fuel cost, operation and management (O\&M) cost, and decommissioning and decontamination (D\&D) cost for each scenario was calculated by considering the mean value from Table 9 . Table 10 shows the breakdown of all the cost elements for two direct disposal and six Pyro-SFR based fuel cycle scenarios. It is noted that the O\&M cost and D\&D cost are assumed to be calculated as $4 \%$ and $8 \%$ of overnight cost of reactors in each scenario in accordance with OECD/NEA studies [37]. In case of scenarios SC-1 and SC-2, the most affecting cost elements are PWR overnight cost followed by PWR fuel, PWR SNF disposal, PWR D\&D, PHWR fuel cost, and others. The potential of continued operation of NPPs (PWRs and PHWRs) in scenario SC-2 up to an additional twenty years increases the total cost by $20.51 \%$. However, the benefit of additional electricity generation to be obtained from continued operation of NPPs (PWRs and PHWRs) increases by $40 \%$.

As far as Pyro-SFR scenarios are concerned, overnight cost followed by the fuel cost of SFRs in each scenario contributes most in the overall Pyro-SFR energy systems. From scenarios SC-3 to SC-8, it is noted that each scenario has advantages and disadvantages in terms of cost, electricity generation, and the minimum number of SFRs needed. For example, if it is decided to transmute all TRUs without 
TABLE 10: Overall scenario cost (unit: M\$ based on 2016 USD).

\begin{tabular}{|c|c|c|}
\hline Case I & SC-1 & SC-2 \\
\hline Number of PWRs & 32 & 32 \\
\hline Number of PHWRs & 4 & 4 \\
\hline PWR overnight cost & 171,955 & 171,955 \\
\hline PHWR overnight cost & 3,329 & 3,329 \\
\hline PWR O\&M cost & 6,878 & $9,604^{*}$ \\
\hline PWR D\&D cost & 13,756 & $19,208^{*}$ \\
\hline PHWR O\&M cost & 133 & $218^{*}$ \\
\hline PHWR D\&D cost & 266 & $435^{*}$ \\
\hline PWR SNF disposal & 39,046 & 54,215 \\
\hline PHWR SNF disposal & 5,799 & 9,497 \\
\hline PWR fuel cost ${ }^{* *}$ & 86,435 & 120,012 \\
\hline PHWR fuel cost** & 14,648 & 23,990 \\
\hline Total PWR cost & 318,071 & 374,995 \\
\hline Total PHWR cost & 24,175 & 37,470 \\
\hline Overall scenario cost & 342,246 & 412,465 \\
\hline PWR electricity generation (TWh) & 12,380 & 17,183 \\
\hline PHWR electricity generation (TWh) & 672 & 1,099 \\
\hline Overall electricity generation (TWh) & 13,052 & 18,282 \\
\hline Case II & SC-3 & $\mathrm{SC}-4$ \\
\hline Minimum number of SFRs needed & 14 & 24 \\
\hline Operating lifetime of SFR (y) & 60 & 60 \\
\hline SFR overnight cost & 51,304 & 87,949 \\
\hline SFR O\&M cost & 2,052 & 3,518 \\
\hline SFR D\&D cost & 4,104 & 7,036 \\
\hline SFR fuel cost ${ }^{* *}$ & 24,402 & 77,920 \\
\hline Overall scenario cost & 82,080 & 176,796 \\
\hline SFR electricity generation (TWh) & 4,014 & 6,870 \\
\hline Case III & SC-5 & SC-6 \\
\hline Minimum number of SFRs needed & 20 & 34 \\
\hline Operating lifetime of SFR (y) & 60 & 60 \\
\hline SFR overnight cost & 73,291 & 124,595 \\
\hline SFR O\&M cost & 2,932 & 4,984 \\
\hline SFR D\&D cost & 5,863 & 9,968 \\
\hline SFR fuel cost ${ }^{* *}$ & 34,861 & 110,387 \\
\hline Overall scenario cost & 117,257 & 250,461 \\
\hline SFR electricity generation (TWh) & 5,734 & 9,732 \\
\hline Case IV & SC-7 & SC-8 \\
\hline Minimum number of SFRs needed & 15 & 25 \\
\hline Operating lifetime of SFR (y) & 80 & 80 \\
\hline SFR overnight cost & 54,968 & 91,614 \\
\hline SFR O\&M cost & 2,932 & 4,886 \\
\hline SFR D\&D cost & 5,863 & 9,772 \\
\hline SFR fuel cost ${ }^{* *}$ & 34,861 & 108,222 \\
\hline Overall scenario cost & 98,865 & 214,896 \\
\hline SFR electricity generation (TWh) & 5,734 & 9,541 \\
\hline
\end{tabular}

O\&M: operation and management; D\&D: decommissioning and decontamination; TWh: terawatt hours. ${ }^{*} \mathrm{O} \& \mathrm{M}$ and $\mathrm{D} \& \mathrm{D}$ costs in SC-2 are derived from SC-1 for 20 years of a more operational period. ${ }^{* *}$ PWR/PHWR/SFR fuel cost represents the total cost of the fuel for the specified type of nuclear reactor. extending the operational lifetime of PWRs and PHWRs, SC3 and SC- 4 can be adopted for short- and long-term transmutation of TRUs, respectively, considering the cost and electricity generation. The major difference between the two is that SC-3 requires a shorter time and a smaller number of SFRs, eventually thus almost twice economical as compared with SC-4. However, the electricity generation from SC- 4 is $41.5 \%$ higher as compared with SC-3, which can be regarded as a long-term benefit. In Case III and Case IV, similar analysis can be applicable in terms of scenario cost, minimum number of SFRs needed, and electricity generation in each scenario.

Scenarios SC-7 and SC-8 reflect the extended operational lifetime of 20 years for all SFRs in order to transmute all TRUs being generated from SC-2. Upon comparison of Case III scenarios with Case IV scenarios, we found out that the total electricity generation from SC-5 and SC-7 is almost the same but SC-7 is more economical compared with SC-5, as it transmutes the same amount of TRUs with fewer units of SFRs while producing the same amount of electricity. In case of scenarios SC-6 and SC-8, relative electricity benefits between the two differ by $1.97 \%$, as SC- 8 generates $98.03 \%$ electricity comparable to SC-6, but in terms of cost and transmutation of TRUs from SC-2, SC-6 is 1.2 times more expensive and requires $26.4 \%$ more SFRs as compared with SC-8.

It is noted that Table 10 should not be used for the direct comparison between direct disposal and Pyro-SFR scenarios because each of SC-1 and SC-2 is a complete nuclear energy system while Pyro-SFR scenarios are just part of the whole nuclear energy system. Pyro-SFR scenarios directly depend on PWR and PHWR fuel cycle because TRUs from SC-1 and SC-2 are the only source of fuel for Pyro-SFR fuel cycle scenarios $[13,31]$.

\subsubsection{Comparison of Direct Disposal and Closed Nuclear} Energy System Cost. In order to compare the economic feasibilities of direct disposal and Pyro-SFR closed fuel cycle, the total scenario costs for Pyro-SFR fuel cycles in Table 10 should be further extended by adding the cost of the front part of the fuel cycle (e.g., existing PWRs and PHWRs fuel cycles).

For the optimized strategy of closed nuclear energy system, scenarios were selected from Table 10 based on the minimum ratio of cost to electricity generation and maximum transmutation of TRUs with the minimum number of SFRs for maximum benefits. For example, scenario SC-2 is chosen as a representative direct disposal scenario because of its higher electricity generation with a smaller increase of cost for continued operation of NPPs (PWRs and PHWRs). Secondly, in order to close the nuclear fuel cycle, scenarios SC-7 and SC-8 were chosen from six Pyro-SFR scenarios because of the minimum number of SFRs to be deployed for the transmutation of larger amounts of TRUs from SC-2 with a lower ratio of cost to electricity generation and higher electricity generation compared with other Pyro-SFR scenarios.

The nuclear energy system cost (i.e., the total costs including overnight cost, fuel cost, O\&M cost, D\&D cost, and SNF disposal cost, if any) for closed fuel cycle is calculated by the combination of scenarios (i.e., CFC-1 by combining SC- 2 and SC-7 and CFC- 2 by combining SC-2 and SC-8) as shown in Table 11. Two of the most affecting factors (i.e., 
TABLE 11: Estimation of closed nuclear energy system costs (unit: M\$ based on 2016 USD).

\begin{tabular}{lcc}
\hline Parameters & CFC-1 & CFC-2 \\
\hline Number of PWRs & 31 & 31 \\
Number of PHWRs & 4 & 4 \\
Minimum number of SFRs needed & 25 & 15 \\
SFR's CR & 0.6 & 0.46 \\
Operating lifetime (PWR/PHWR/SFR) & $60 / 50 / 80$ & $60 / 50 / 80$ \\
SFR overnight cost & 91,614 & 54,968 \\
PWR overnight cost & 171,955 & 171,955 \\
PHWR overnight cost & 3,329 & 3,329 \\
SFR fuel cost & 108,222 & 34,861 \\
PWR fuel cost & 120,012 & 120,012 \\
PHWR fuel cost & 23,990 & 23,990 \\
SFR O\&M cost & 4,886 & 2,932 \\
PWR O\&M cost & 9,604 & 9,604 \\
PHWR O\&M cost & 218 & 218 \\
SFR D\&D cost & 9,772 & 5,863 \\
PWR D\&D cost & 19,208 & 19,208 \\
PHWR D\&D cost & 435 & 435 \\
Closed nuclear energy system cost & 563,246 & 447,376 \\
Electricity generation (TWh) & 28,841 & 25,035 \\
\hline
\end{tabular}

CFC: closed fuel cycle.

PWR overnight and fuel costs) in SC-2 and two of the most affecting factors in SC-7 and SC-8 (i.e., SFR overnight and fuel costs) consist of the most dominant cost elements in CFC-1 and CFC-2, respectively, as listed in Table 11. It can be noted that CFC-2 is $20.57 \%$ cheaper than CFC-1 with only $13.19 \%$ less electricity generation because a smaller number of SFRs are needed for CFC-2. From Table 11, we can see that both CFC-1 and CFC-2 can be utilized for different objectives. That is, CFC-2 can be chosen for fast transmutation of TRUs with the minimum number of SFRs. On the other hand, CFC-1 can be selected for extended transmutation of TRUs with a comparatively higher number of SFRs, which generates $13.19 \%$ more electricity.

Figure 7 shows the statistics from the Monte Carlo simulation with the assumption that each parameter of direct disposal and closed nuclear energy system has a triangular distribution. A series of Monte Carlo simulations were carried out using 50,000 samples, and the total generation costs for SC-1, SC-2, CFC-1, and CFC-2 are calculated to be $24.497 \pm 2.995(1 \sigma), 21.382 \pm 2.318,19.220 \pm 1.817$, and $17.620 \pm$ $1.785 \$ / \mathrm{kWh}$, respectively. Scenarios SC-1 and SC-2, which are a combination of PWR and PHWR nuclear energy systems, are being considered as a single integrated direct disposal nuclear energy system; hence, the cost incurred at any point in SC- 1 and SC-2 should be normalized with the electricity produced throughout SC-1 and SC-2. Similarly, fuel cycle with recycling of TRUs is considered as a single integrated technology; therefore, the cost incurred at any point in the CFC- 1 and CFC-2 is normalized across the electricity produced throughout the whole closed fuel cycle [35].

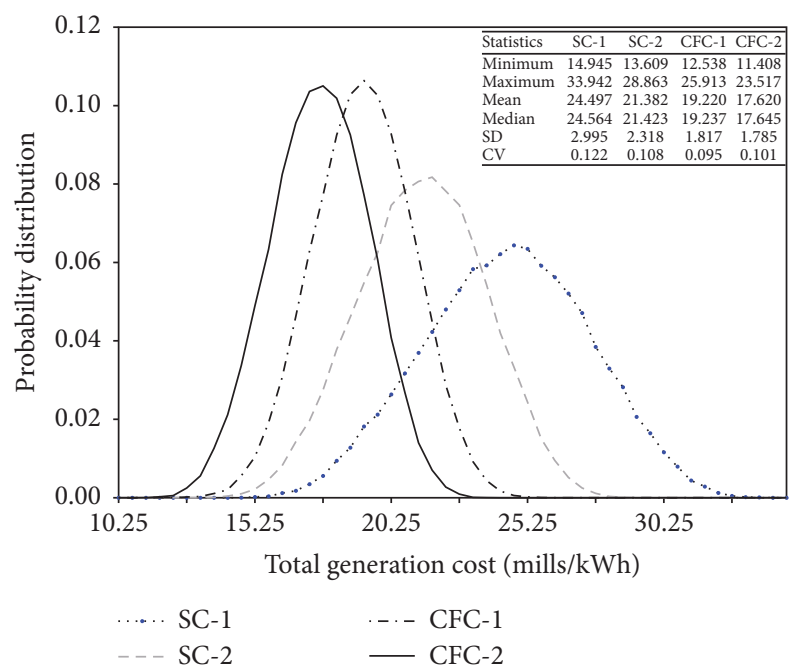

Figure 7: Probabilistic estimation of the total generation cost for direct disposal and closed nuclear energy systems using 50,000 samples.

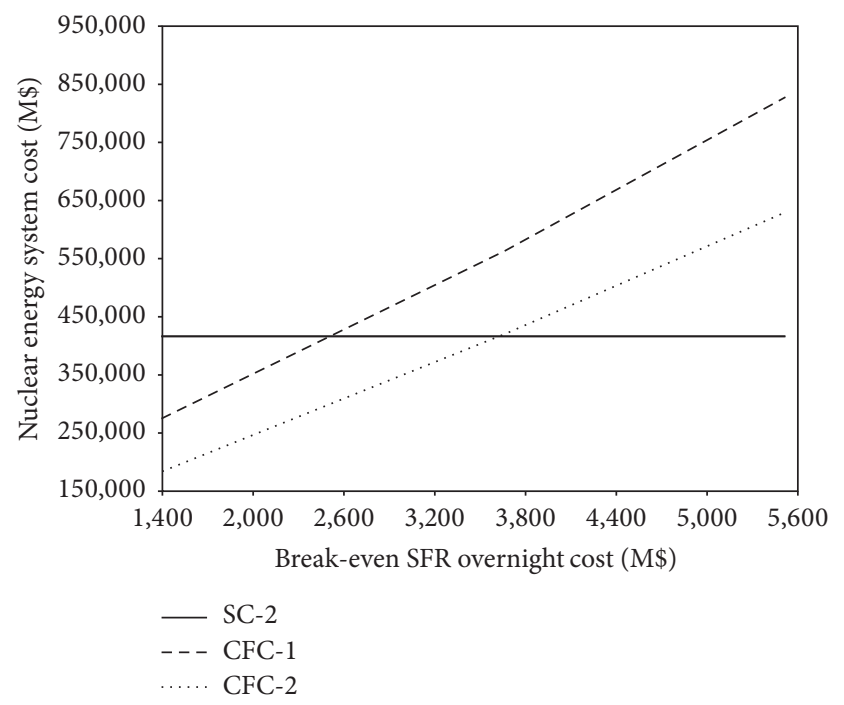

FIGURE 8: Relation between the break-even points of unit SFR overnight cost with CFC-1, CFC-2, and SC-2.

3.4.5. Break-Even SFR Overnight Cost. In order to determine a specific condition under which the Pyro-SFR closed nuclear energy system shows positive economic feasibility over direct disposal option, a break-even point of SFR overnight cost is estimated. As shown in Figure 8, the break-even SFR overnight cost per reactor for CFC-1 and CFC- 2 is calculated to be $M \$ 2,570$ and $M \$ 3,450$, respectively. It can be said that the closed nuclear energy system CFC-1 or CFC-2 is more economically feasible compared to direct disposal scenario SC-2 if SFR overnight cost falls below the break-even point as calculated in the figure.

3.4.6. Sensitivity Analysis for Closed Fuel Cycles. Sensitivity analysis has been carried out to analyze the cost elements in closed fuel cycle that may affect the nuclear energy system 


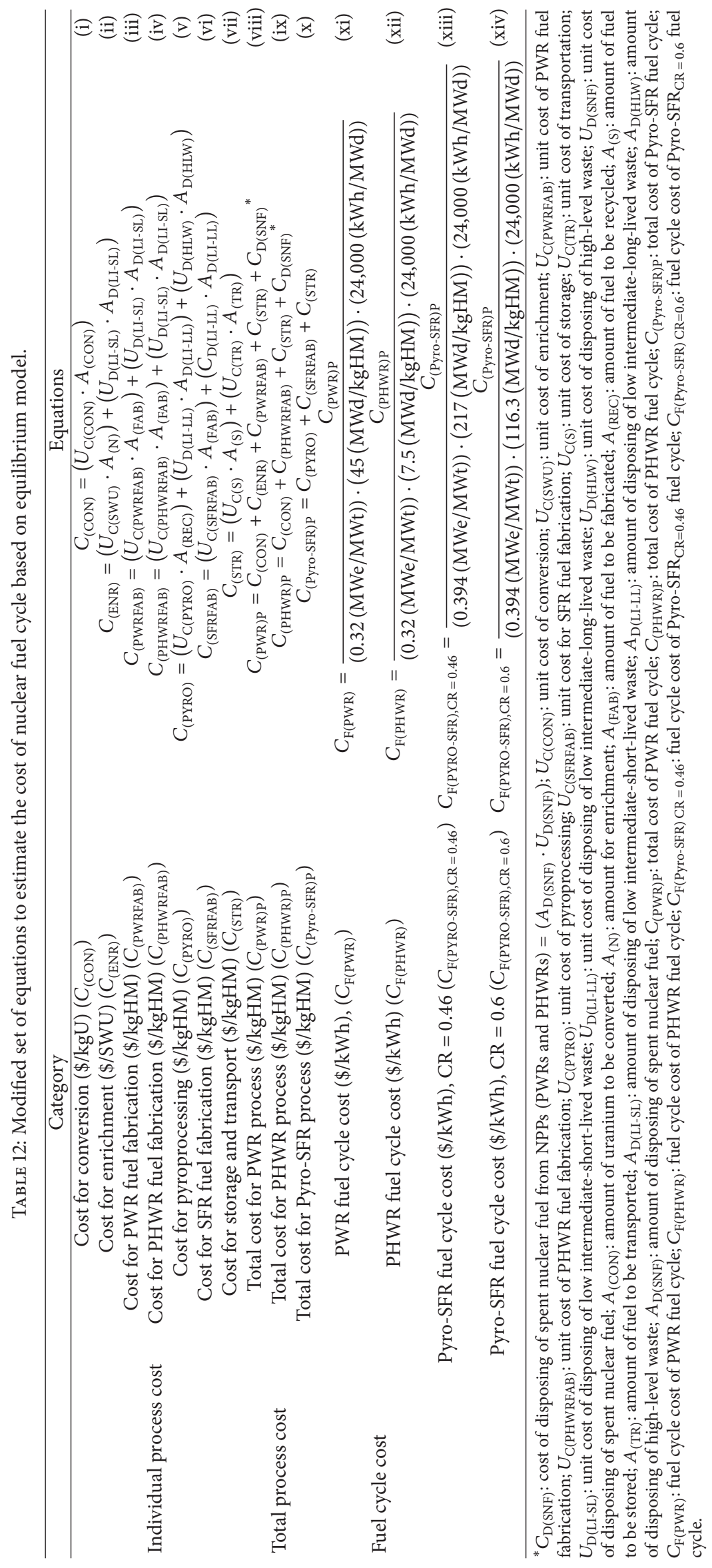




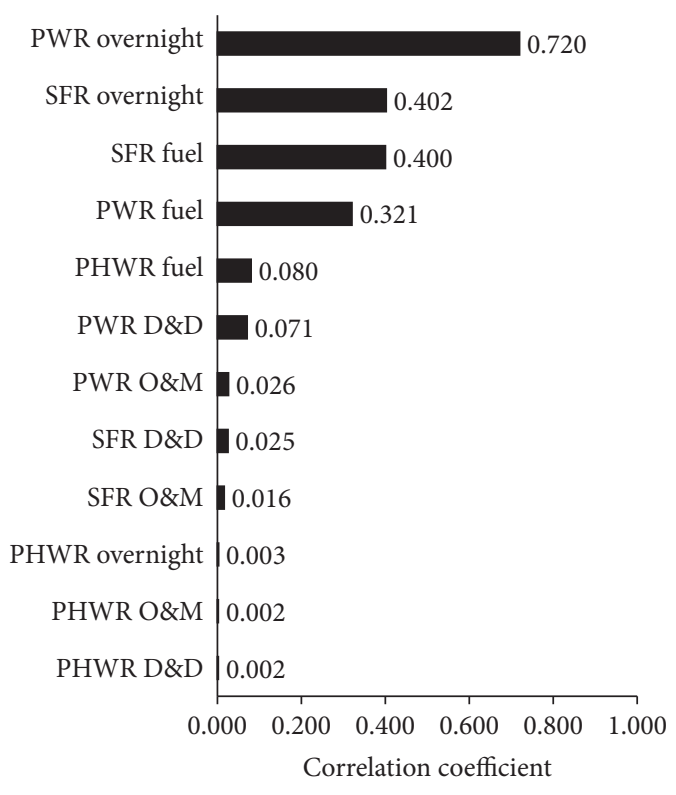

(a)

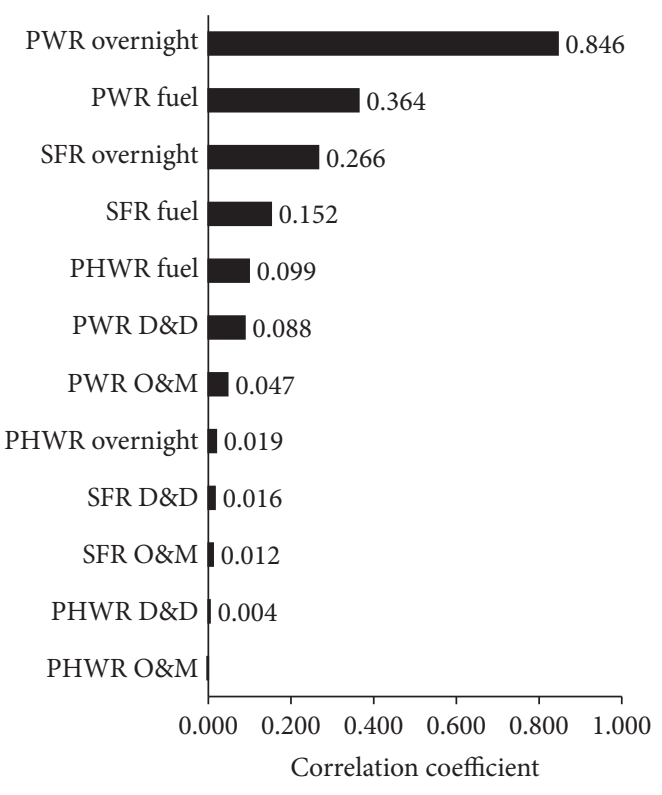

(b)

FIGURE 9: Sensitivity of cost elements in closed fuel cycle nuclear energy systems: (a) CFC-1 and (b) CFC-2.

cost by using Crystal Ball software. Crystal Ball computes the sensitivity by computing rank correlation coefficients. Correlation coefficients measure the strength of the linear relationship among the parameters in CFC-1 and CFC-2. A parameter having a higher correlation coefficient value affects more the total cost of closed nuclear energy system.

Figure 9 shows that the five most affecting factors in CFC1 and CFC-2 are the PWR overnight cost, SFR overnight cost, SFR fuel cost, PWR fuel cost, and PHWR fuel cost. As mentioned, it is noted that, even though sensitivity for direct disposal energy system is not conducted, the relative sensitivity of cost elements in SC- 1 and SC-2 can be calculated by taking into account the cost parameters related to PWR and PHWRs only from Figure 9.

\section{Conclusion}

The total inventory of SNF to be produced from all thirtysix units of NPPs that are in operation or will be deployed by 2029 is estimated to be $41,718 \mathrm{MTU}$, in accordance with the national policy of the Korean government in 2016. It is also found that the continued operation of NPPs may increase the national inventory of SNF by $47 \%$ (i.e., up to 61,232 MTHM). In this study, it is reconfirmed that the nominal radiotoxicity index of the long-lived TRUs in the SNF will decrease down to the level of natural uranium at least after 200,000 years of radioactive decay as reportedly known.

Based on a series of reasonable assumptions derived from past experience of nuclear power development in Korea and the government's future plan, the minimum number of SFRs required to transmute all TRUs is calculated to be 14 to 34 , and the full transmutation of TRUs is expected to be completed in 2109 to 2153 . The key technical elements determining the optimized strategy for SFR deployment are the design features of SFR (e.g., conversion ratio, burnup), the deployment rate of SFRs, and the target year for completion of transmutation of TRUs.

A systematic stepwise procedure to conduct the probabilistic economic analysis of nuclear energy system is proposed in this study (see Figure 2). From balanced comparison of the whole cost and all benefits from direct disposal and Pyro-SFR recycling options, the total generation costs for direct disposal and Pyro-SFR nuclear energy systems are estimated to be 13.60 33.94 mills/kWh and 11.40 25.91 mills/ $\mathrm{kWh}$, respectively. It is concluded that the relative feasibility of each scenario can be assessed based upon the three factors: lower ratio of cost to electricity generation, transmutation of more TRUs with minimum units of SFRs, and higher generation of electricity in order to formulate the optimum closed nuclear energy system.

As a result of the sensitivity analysis, the most affecting parameters to the nuclear energy system costs of the closed fuel cycle are as follows in order of contribution: PWR overnight cost, PWR fuel cost, SFR overnight cost, and SFR fuel cost. Since the overnight cost and the fuel cost for PWR are almost invariable in the commercialized nuclear energy system in Korea, however, the overnight cost of the SFR still under development will be the most uncertain cost element to the economics of the closed nuclear energy system. It is also pointed out that the economic feasibility of the closed nuclear energy system is higher than the direct disposal option as long as the SFR overnight cost per reactor can be kept below $M \$ 2,570$ or $M \$ 3,450$ (i.e., the break-even reactor cost) depending upon the design features of the SFR such as conversion ratio.

It is expected that the results of this study on the quantitative and economic feasibilities of direct disposal and Pyro-SFR fuel cycle options can be used as a comprehensive 
reference for the systematic decision-making on the direction of the future nuclear energy system in Korea, which is planned to be made around 2020.

\section{Conflicts of Interest}

All authors declare no conflicts of interest regarding the publication of this paper.

\section{Acknowledgments}

This work was supported by "Human Resources Program in Energy Technology" of the Korea Institute of Energy Technology Evaluation and Planning (KETEP), granted financial resource from the Ministry of Trade, Industry \& Energy, Republic of Korea (no. 20164030200990).

\section{References}

[1] Korea Institute of Nuclear Safety (KINS), Review Report on the Quarterly Reports form Nuclear Business Operators Regarding Radiation Safety for the First Quarter of 2016, KINS, 2016.

[2] Ministry of Trade and Industry and Energy (MOTIE), "The 7th Basic Plan on Electricity Demand and Supply," Notice No. 2015403, MOTIE, 2015.

[3] Ministry of Trade and Industry and Energy (MOTIE), "Basic Plan for High Level Radioactive Waste Management," MOTIE, 2016.

[4] Ministry of Science and ICT and Future Planning (MSIP), Strategy for Technical Development and Demonstration of Future Nuclear Energy System, MSIP, 2016.

[5] The government of the Republic of Korea and C. Pinel, "Korean Fifth National Report under the Joint Convention on the Safety of Spent Fuel Management and on the Safety of Radioactive Waste Management," 2014.

[6] J. Kang, "Alternatives for additional spent fuel storage in South Korea," Science \& Global Security, vol. 10, no. 3, 2002.

[7] J. Park, "Status of the korean high-level radioactive waste management program," in in proceedings of ANS Conference International High-level Radioactive Waste Management (IHLRWM 2017), Charlotte, NC, USA, April 9-13, 2017.

[8] Y. I. Kim, S. G. Hong, and D. Hahn, "SFR deployment strategy for the re-use of spent fuel in Korea," Nuclear Engineering and Technology, vol. 40, no. 6, pp. 517-526, 2008.

[9] J. Kang, "The ROK's nuclear energy development and spent fuel management plans," NAPSNet Special Reports, June 17, 2014, https://nautilus.org/napsnet/napsnet-special-reports/theroks-nuclear-energy-development-and-spent-fuel-managementplans/.

[10] C. Braun and R. Forrest, "Considerations regarding ROK spent nuclear fuel management options," Nuclear Engineering and Technology, vol. 45, no. 4, pp. 427-438, 2013.

[11] B. H. Park, F. Gao, E.-H. Kwon, and W. I. Ko, "Comparative study of different nuclear fuel cycle options: Quantitative analysis on material flow," Energy Policy, vol. 39, no. 11, pp. 69166924, 2011.

[12] S. K. Kim, W. I. Ko, S. R. Youn, and R. X. Gao, "Nuclear fuel cycle cost estimation and sensitivity analysis of unit costs on the basis of an equilibrium model," Nuclear Engineering and Technology, vol. 47, no. 3, pp. 306-314, 2015.
[13] F. Gao and W. I. Ko, "Dynamic analysis of a pyroprocessing coupled SFR fuel recycling," Science and Technology of Nuclear Installations, vol. 2012, Article ID 390758, pp. 1-10, 2012.

[14] W. I. Ko and F. Gao, "Economic analysis of different nuclear fuel cycle options," Science and Technology of Nuclear Installations, vol. 2012, Article ID 293467, pp. 1-10, 2012.

[15] S. K. Kim, W. I. Ko, and Y. H. Lee, "Economic viability of metallic sodium-cooled fast reactor fuel in Korea," Science and Technology of Nuclear Installations, vol. 2013, Article ID 412349, pp. 1-10, 2013.

[16] S. K. Kim, W. I. Ko, and Y. H. Lee, "Economic assessment of alternative Pyro-SFR nuclear fuel cycle in Korea," Progress in Nuclear Energy, vol. 66, pp. 124-132, 2013.

[17] R. Gao, S. Choi, W. Il Ko, and S. Kim, "Economic potential of fuel recycling options: A lifecycle cost analysis of future nuclear system transition in China," Energy Policy, vol. 101, pp. 526-536, 2017.

[18] S. Choi, H. J. Lee, and W. I. Ko, "Dynamic analysis of oncethrough and closed fuel cycle economics using Monte Carlo simulation," Nuclear Engineering and Design, vol. 277, pp. 234247, 2014.

[19] Ministry of Knowledge Economy (MKE), Cost estimation of interim storage for spent fuels, Ministry of Knowledge Economy of Republic of Korea, Seoul, Korea, 2012.

[20] Ministry of Knowledge Economy (MKE), Ministry of Knowledge Economy Cost Estimation of Final Disposal for Spent Fuels, Ministry of Knowledge Economy of Republic of Korea, Seoul, Korea, 2012.

[21] C. S. Rim, "Korean nuclear fuel program," Journal of Nuclear Science and Technology, vol. 35, no. 7, pp. 467-472, 1998.

[22] F. Fiori and Z. Zhou, "Sustainability of the Chinese nuclear expansion: The role of ADS to close the nuclear fuel cycle," Progress in Nuclear Energy, vol. 83, pp. 123-134, 2015.

[23] P. Villalibre, E. Haas, H. Khartabil, S. Kim, A. Korinny, V. Usanov et al., "Technical Progress in INPRO activities on Modelling and Innovation," in INPRO Group Department of Nuclear Energy, International Atomic Energy Agency (IAEA), Vienna, Austria.

[24] F. Fiori and Z. Zhou, "Sustainability of the Chinese nuclear expansion: Natural uranium resources availability, Pu cycle, fuel utilization efficiency and spent fuel management," Annals of Nuclear Energy, vol. 83, pp. 246-257, 2015.

[25] IAEA, "Nuclear Fuel Cycle Simulation System (VISTA)," in IAEA TECDOC-1535, Vienna, Austria, February 2007.

[26] IAEA, Nuclear Fuel Cycle Simulation System (NFCSS) user manual, IAEA, Vienna, Austria, December 2011.

[27] Nuclear Power in South Korea, "World Nuclear Association," last updated February 2017, http://www.world-nuclear.org/ information-library/country-profiles/countries-o-s/southkorea.aspx.

[28] Y. Arafat et al., "Radiotoxicity characterization of hlw from reprocessing of Uranium-based and Thorium- based fuel," in WM2011 Conference, Phoenix, Arizona, February 27 - March 3, 2011.

[29] The Korean Academy of Science and Technology, "Management of high level nuclear wastes," Research Reports Series No. 54, 2009.

[30] International Commission on Radiological Protection, "Compendium of dose coefficients based on ICRP publication 60, ICRP publication no. 119," Annals of ICRP, vol. 41, no. S1, 2012. 
[31] C. Jeong and H. Choi, "Dynamic modeling and analysis of alternative fuel cycle scenarios in Korea," Nuclear Engineering and Technology, vol. 39, no. 1, pp. 85-94, 2007.

[32] Y. K. Lee and M. H. Kim, "Recycling option search for a 600MWe sodium-cooled transmutation fast reactor," Nuclear Engineering and Technology, vol. 47, no. 1, pp. 47-58, 2015.

[33] S. G. Hong, S. J. Kim, Y. I. Kim, and D. Hahn, "600 MWe Sodium cooled fast reactor core designs for efficient TRU transmutation," in in Proceedings of the The Korean Nuclear Society Spring Meeting, pp. 26-27, Republic of Korea, May 2006.

[34] Korea Atomic Energy Research Institute (KAERI), "Development of Sodium Cooled Fast Reactor Core Concept for Maximizing TRU Burning Rate," KAERI/CN-2209/2015, Daejeon, Korea, 2015.

[35] G. De Roo and J. E. Parsons, "A methodology for calculating the levelized cost of electricity in nuclear power systems with fuel recycling," Energy Economics, vol. 33, no. 5, pp. 826-839, 2011.

[36] M. Bunn, B. van der, J. P. Zwaan, and S. Fetter, "The economics of reprocessing vs. direct disposal of spent nuclear fuel," Tech. Rep. DE-FG-26-99FT4028, 2003.

[37] OECD/NEA, "Advance nuclear fuel cycle and radioactive waste management,” Tech. Rep. 5990.OECD, Nuclear Energy Agency (NEA), Paris, France, 2006.

[38] Los Alamos National Laboratory (LANL), "Production and Measurement of Minor Actinides in the Commercial Fuel Cycle," Tech. Rep., 1997, LA-13248-MS/UC-700/1997.

[39] WISE Uranium Project, "Uranium toxicity," 2017, http://www .wise-uranium.org/utox.html.

[40] OECD/NEA, The economics of the back end of the nuclear fuel cycle, OECD, Nuclear Energy Agency (NEA), Vienna, Austria, 2013.

[41] Smith School of Enterprise and the Environment (SSEE), A low carbon nuclear future: Economic assessment of nuclear materials and spent nuclear fuel management in the UK, Oxford, United Kingdom, March 2011.

[42] D. Shropshire, K. A. Williams, W. B. Boore et al., "Advanced fuel cycle cost basis," Tech. Rep. INL/EXT-07-12107, Idaho National Laboratory (INL), 2007.

[43] D. Shropshire, E. A. Hoffman, J. D. Smith et al., "Advanced Fuel Cycle Economic Analysis of Symbiotic Light-Water Reactor and Fast Burner Reactor Systems," Idaho National Laboratory (INL), no. INL/EXT-09-15254, 2009.

[44] G. Francesco and B. Dixon, "Nuclear energy system cost modeling actinide and fission product partitioning and transmutation," in Proceedings of the 12th Information Exchange Meeting on Actinide and Fission Product Partitioning and Transmutation, Idaho National Laboratory (INL), September 2012.

[45] G. E. Evans and B. Jones, "The application of Monte Carlo simulation in finance, economics and operations management," in Proceedings of the 2009 WRI World Congress on Computer Science and Information Engineering, CSIE 2009, pp. 379-383, usa, April 2009.

[46] E. Parent, Nuclear fuel cycles for mid-century deployment, Massachusetts Institute of Technology, September 2003. 


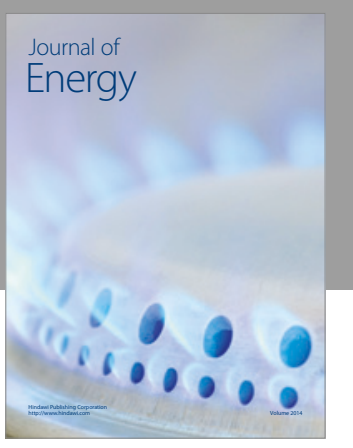

Journal of

Industrial Engineering
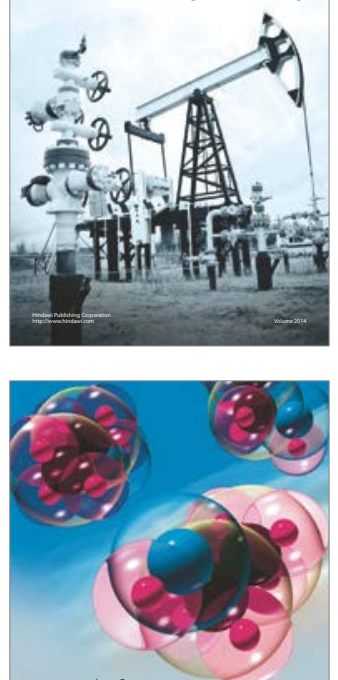

Fuels
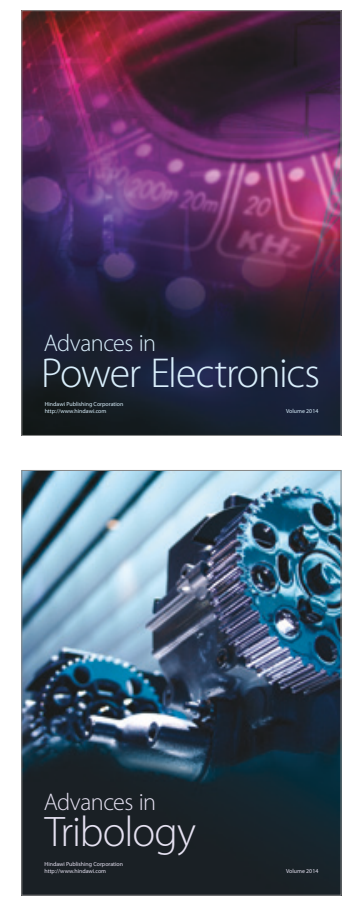
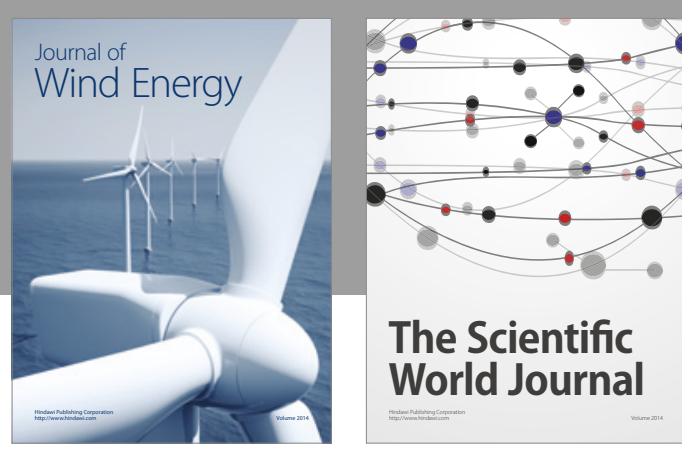

The Scientific World Journal
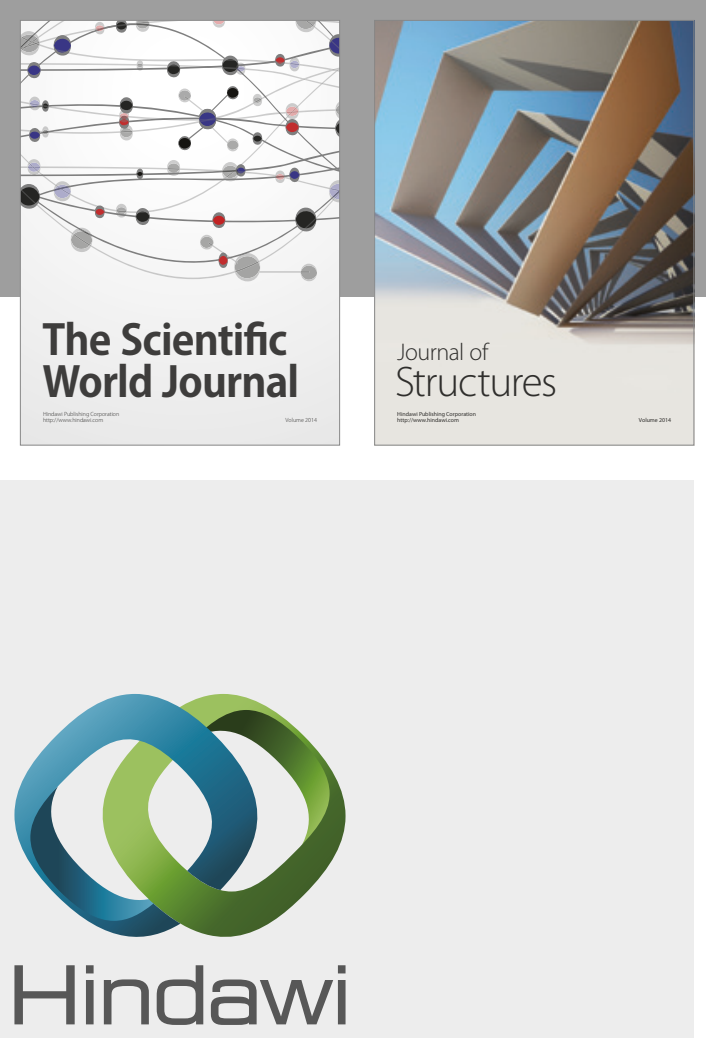

Submit your manuscripts at

https://www.hindawi.com
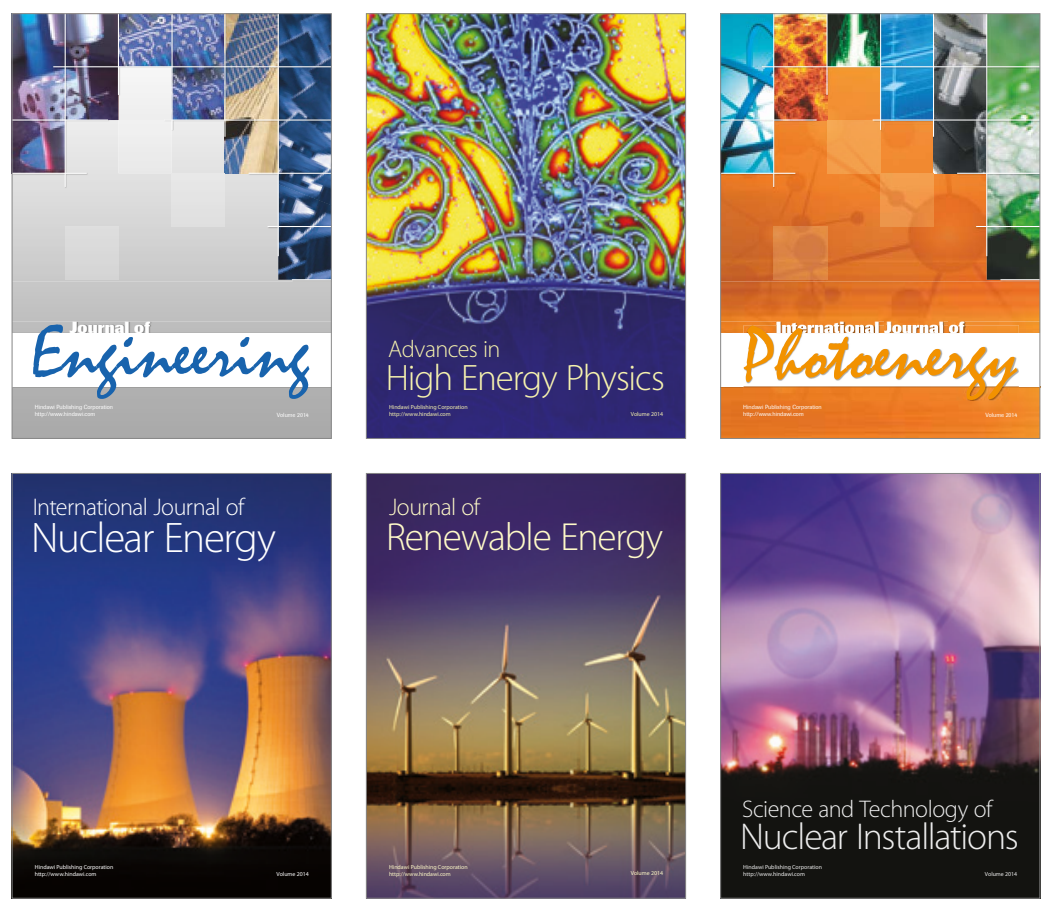

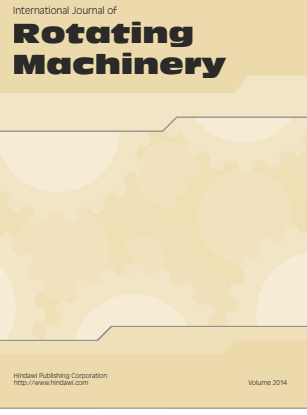

Journal of

Petroleum Engineering

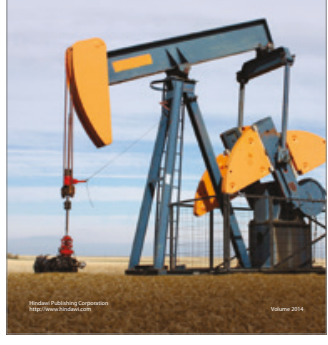

Journal of
Solar Energy
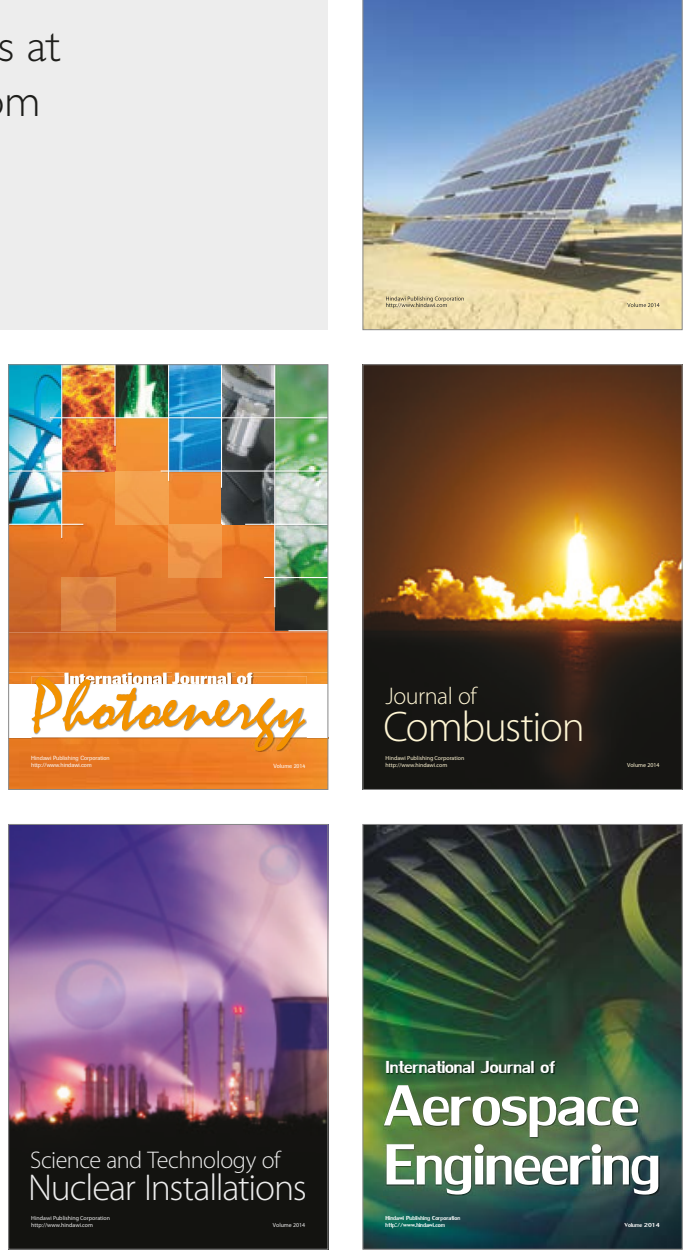